\title{
La evolución tectónica y magmática cenozoica del suroeste de México: avances y problemas de interpretación
}

\author{
Dante Jaime Morán-Zenteno ${ }^{1, *}$, Mariano Cerca ${ }^{1,2}$, John Duncan Keppie ${ }^{1}$ \\ ${ }^{1}$ Instituto de Geología, Universidad Nacional Autónoma de México, \\ Ciudad Universitaria, Delegación Coyoacán, 04510, México D.F. \\ ${ }^{2}$ Centro de Geociencias, Universidad Nacional Autónoma de México, \\ Campus Juriquilla, Querétaro, Qro. 76230, México. \\ *dantez@servidor.unam.mx
}

\section{Resumen}

Los avances recientes en el conocimiento de la estructura y la estratigrafía cenozoicas del sur de México revelan una evolución caracterizada por eventos de deformación orogénica iniciados en el Cretácico Tardío, seguidos por episodios de truncamiento de la margen continental y extinción gradual del magmatismo de arco en la Sierra Madre del Sur, antes del desarrollo del Faja Volcánica Transmexicana.

La deformación orogénica que se inició en el Cretácico Tardío es contemporánea y de orientación similar a la orogenia Laramide. Se caracteriza por un acortamiento E-W con vergencia al este y una migración de la deformación también hacia el este. Los modelos que relacionan el origen de la deformación laramídica a la disminución en el ángulo de subducción de la placa de Farallón no pueden aplicarse en esta región, en virtud de la presencia de magmatismo de arco en posiciones relativamente cercanas a la paleotrinchera durante el Paleoceno y el Eoceno temprano. La posibilidad de que esta deformación se deba a la colisión de un arco insular en la margen continental occidental no parece ser viable por la ausencia de rasgos o asociaciones petrológicos típicos de una sutura por el cierre de una cuenca oceánica.

El patrón de extinción general del magmatismo desde el Cretácico Tardío y Paleoceno en Colima y Jalisco, hasta el Mioceno medio en la parte central y suroriental de Oaxaca, presenta a la luz de los datos geocronológicos recientes, variaciones que rompen con un esquema simple de extinción hacia el ESE. El plutonismo del Maastrichtiano-Paleoceno temprano reconocido en la región del bloque Jalisco y Manzanillo coexistió con un episodio magmático de la misma edad en la parte central de la Sierra Madre del Sur, para el cual se han reportado algunas afinidades adakíticas. El magmatismo en el intervalo entre el Paleoceno y el Eoceno medio parece estar concentrado en la zona de la Presa del Infiernillo, aunque existen centros aislados en zonas como Taxco o el oriente del bloque Jalisco. Finalmente, el eje principal del magmatismo entre el Eoceno medio y el Oligoceno, se desarrolló a lo largo de la margen continental actual pero también hubo considerable volcanismo en una franja ubicada a $200 \mathrm{~km}$ hacia el interior del continente. En general, los caracteres geoquímicos de este magmatismo indican una baja asimilación de la corteza continental antigua.

Para el Eoceno e inicios del Oligoceno se han reconocido dos episodios de fallamiento lateral, principalmente izquierdo, que variaron en tiempo y espacio y que activaron fallas de orientación al NW y N-S sucesivamente. Este último conjunto de fallas parece haber sido activo sólo en el norte de la Sierra Madre del Sur, mientras que el primero siguió activo durante el Oligoceno en la margen continental de Oaxaca. El reconocimiento de estos episodios de deformación sugiere que las direcciones de extensión relacionadas al fallamiento lateral variaron de NNW-SSE a NE-SW, y que localmente activaron fallas normales en discontinuidades preexistentes.

Existen todavía problemas fundamentales respecto a la interpretación de los procesos de tectónica de placas que originaron los regímenes de esfuerzos que activaron los diferentes conjuntos de fallas que han sido documentados hasta ahora, así como sobre los factores que causaron los patrones de migración magmática observados. Por una parte, han 
permanecido inciertos algunos de los argumentos sostenidos para postular la presencia del bloque de Chortis, durante el Cenozoico temprano, frente a la actual margen continental del suroeste de México. Por otro lado, los modelos que explican los desplazamientos restringidos con respecto al bloque Maya y sin una yuxtaposición con la margen SW de México, sugieren más bien un truncamiento continental producido esencialmente por erosión por subducción pero dejan abiertas las causas de la migración magmática observada.

Palabras clave: Sur de México, magmatismo de arco, deformación, tectónica, Cenozoico.

\begin{abstract}
Recent advances in the knowledge of the Cenozoic structure and stratigraphy of southern Mexico reveal a geological evolution characterized by Late Cretaceous orogenic deformation, followed by truncation of the continental margin and gradual extinction of arc magmatism in the Sierra Madre del Sur, previous to the onset of magmatism in the TransMexican Volcanic Belt.

Orogenic deformation beginning in the Late Cretaceous was coeval with the Laramide orogeny and displays structures with a similar orientation. Deformation consisted of $E$-W shortening with a general eastward vergence and a well defined migration to the east. Models that associate the origin of Laramide deformation to a decrease in the angle of subduction of the Farallon plate, which was converging in western Mexico, cannot be applied in this region because of the occurrence of Paleocene to early Eocene arc magmatism near the inferred paleotrench. A possible origin related to the collision of an insular arc against the western margin of Mexico does not seem plausible due the absence of features and petrogenetic associations indicating the closure of an oceanic basin.

In light of recent geochronological data, the general pattern of magmatism extinction, from Late Cretaceous-Paleocene in Colima and Jalisco to the middle Miocene in central and southeastern Oaxaca, presents variations inconsistent with a simple pattern of extinction toward the east-southeast.

Maastrichtian to early Paleocene plutonism recognized in the Jalisco block and Manzanillo areas is contemporaneous with a magmatic episode with some documented adakite affinities in the central part of the Sierra Madre del Sur. Magmatism from the Paleocene to middle Eocene seems to be concentrated in the Presa del Infiernillo area, although isolated centers existed in areas such as Taxco or the eastern Jalisco block. Finally, the main axis of magmatism between the middle Eocene and Oligocene developed along what is now the present day continental margin and also within a band located $200 \mathrm{~km}$ inland. In general, the geochemical characteristics of this magmatism indicate a low degree of continental crustal assimilation.

Two episodes of principally sinistral lateral faulting that activated NW-SE and later N-S oriented faults, with variations in time and space, have been documented during the Eocene and the beginning of the Oligocene. The N-S set of faults was active only in the north of the Sierra Madre del Sur, whereas the activity of the NW-SE set continued during the Oligocene along the Oaxaca continental margin. The recognition of these deformation episodes suggests that extension directions related to lateral faulting changed from NNW-SSE to NE-SW, and locally activated normal displacements on pre-existing discontinuities.

Fundamental problems still exist in the interpretation of the plate tectonic processes that originated the stress regimes acting on the different sets of faults, as well as in the knowledge of the factors influencing the migration of magmatism. Some of the arguments used to postulate the presence of the Chortis block off the southwestern Mexico continental margin during the early Cenozoic are uncertain. On the other hand, models that explain restricted displacements of the Chortis block with respect to the Maya block without juxtaposition with the southwestern margin of Mexico suggest that continental truncation was essentially caused by subduction erosion but leave open the interpretation of the observed magmatic migration.
\end{abstract}

Key words: Southern Mexico, arc-magmatism, deformation, tectonics, Cenozoic.

\section{Introducción}

El conocimiento sobre la estructura y la evolución geológica cenozoica del sur de México ha experimentado progresos significativos durante los últimos años. A pesar de que existen todavía zonas sin una descripción geológica detallada, se han formulado modelos generales de evolución tectónica basados en: (1) observaciones y datos sobre rasgos clave de la corteza continental y, (2) las inferencias sobre la cinemática de las placas tectónicas en el entorno oceánico. Existen todavía importantes controversias sobre aspectos significativos de la evolución geológica de 
esta región, pero la creciente información disponible ha llevado a un nivel más profundo la discusión. Uno de los antecedentes más notables fue la identificación, a partir de la década de los años ochenta, de contrastes petrotectónicos y geocronológicos en las rocas del basamento premesozoico que definen un mosaico de terrenos para esta región (Ortega- Gutiérrez, 1981; Campa y Coney, 1983; Sedlock et al., 1993; Keppie, 2004) (Figura 1). El reconocimiento de este basamento de características contrastantes ha permitido comprender mejor algunos aspectos de la evolución tectónica mesozoica y cenozoica del sur de México. Otros antecedentes importantes son los trabajos en los que se han reconocido y descrito los aspectos generales de la estratigrafía cenozoica en diferentes zonas del sur de México $(i$. e., Fries, 1960, 1966; De Cerna y Fries, 1981; FerrusquíaVillafranca, 1976, 1992; Martiny et al., 2000) y en los que se describen algunos rasgos tectónicos sobresalientes (Ratschbacher et al., 1991; Tolson et al., 1993; Riller et al., 1993; Meschede et al., 1996; Nieto-Samaniego y AlanizÁlvarez, 1995; Alaniz-Álvarez et al., 2002; Cerca et al., 2004). Existen además numerosas contribuciones recientes sobre la geocronología y la geoquímica de las rocas magmáticas cenozoicas en esta región que han permitido definir patrones espacio-temporales de la actividad magmática y algunos de los aspectos petrogenéticos más sobresalientes (i. e., Herrmann et al., 1994; Schaaf et al., 1995; MoránZenteno et al., 1999, 2004; Martiny et al., 2000; Ferrari et al., 1999; Meza-Figueroa et al., 2003; González Partida et al., 2003; Ducea et al., 2004a). Una referencia importante para la interpretación sobre la evolución geológica del sur de México son los estudios sobre rasgos estructurales de la corteza oceánica y su edad, así como la cinemática de las placas en el entorno del sur de México (Figura 1). A partir de esta información se han generado modelos que han causado una influencia importante en las interpretaciones tectónicas cenozoicas para la región (Malfait y Dinkelman, 1972; Anderson y Smith, 1983; Ross y Scotese, 1988; Pindell et al., 1988; Pindell y Barret, 1990; Keppie y Morán -Zenteno, 2005).

Existe un registro amplio de rocas volcánicas y plutónicas para el sur de México, así como una diversidad de secuencias sedimentarias continentales, tanto asociadas a extensión en zonas de fallamiento lateral, como cuencas intermontanas y reactivación de fallas preexistentes. Los procesos de levantamiento y denudación en el sur de México que se iniciaron desde el final del Cretácico han propiciado la acumulación de secuencias continentales, aunque en la mayoría de los casos estas potentes secuencias se conservan sólo localmente.

Los estudios sobre la deformación y el magmatismo del sur de México han mostrado que la región ubicada al sur de la Faja Volcánica Transmexicana (FVTM), aunque comparte algunas características estratigráficas y magmáticas con el norte de México, manifiesta diferencias sobresalientes en su evolución tectónica. Estas diferencias parecen estar definidas principalmente por la influencia de las interacciones tectónicas en la margen del Pacífico, diferentes en el sur de México con respecto a las del norte de México, y por las variaciones en el basamento y en las estructuras tectónicas preexistentes. Aunque no se ha estudiado con detalle, las diferencias en las estructuras durante el emplazamiento de magmas que implican un cambio en el estilo tectónico del sur con respecto al norte de México son evidentes durante el Eoceno u Oligoceno (i.e., Nieto-Samaniego et al., 1999;

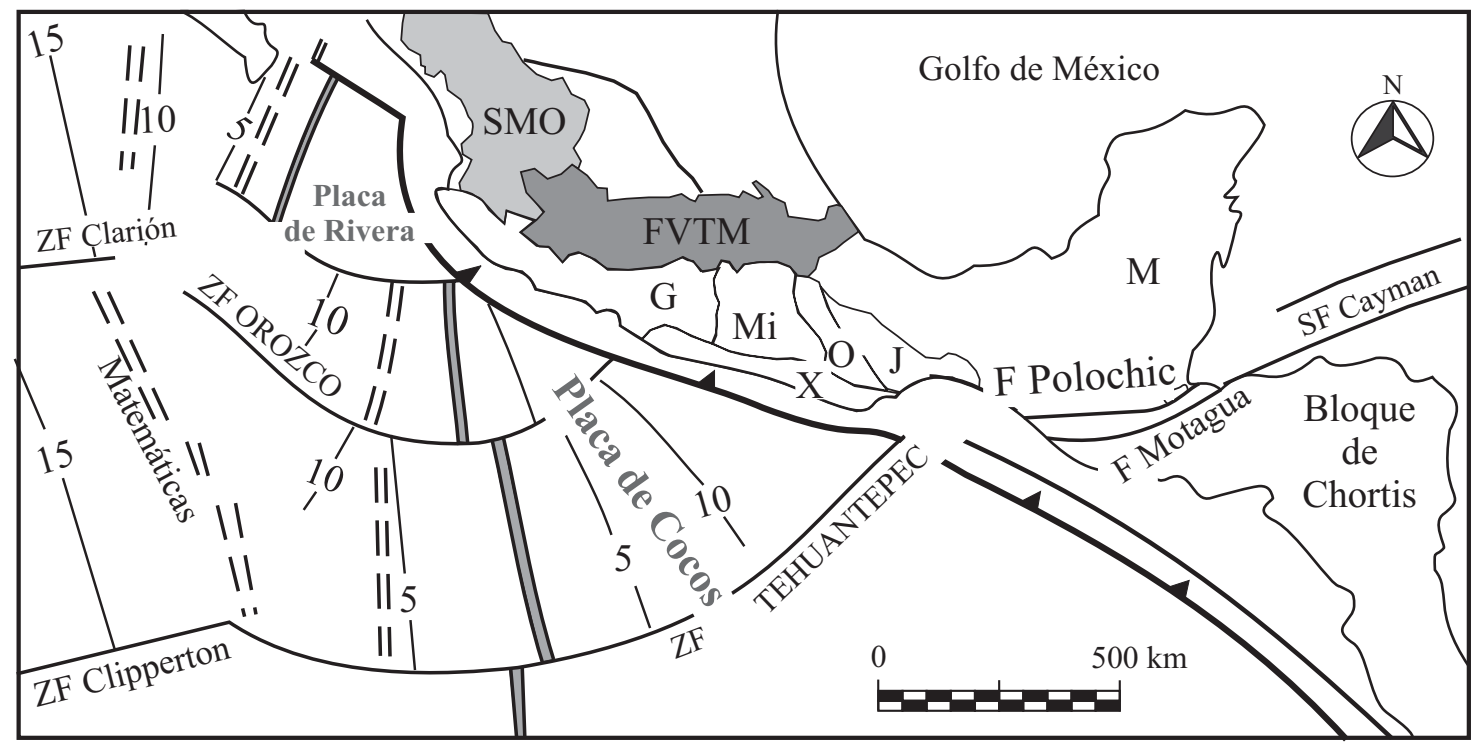

Figura 1. Principales rasgos tectónicos del entorno oceánico actual del sur de México. También se muestra la distribución de los terrenos tectonoestratigráficos pre-cenozoicos (modificado de Campa y Coney, 1983). SMO, Sierra Madre Occidental; FVTM, Faja Volcánica Transmexicana; G, terreno Guerrero; Mi, terreno Mixteca; O, terreno Oaxaca; J, terreno Juárez; X, terreno Xolapa; M, terreno Maya. ZF, zona de fractura. 
Ferrari et al., 2002; Alaniz-Álvarez et al., 2002; MoránZenteno et al., 2004).

En este trabajo se presenta una revisión sobre el estado actual del conocimiento acerca de la evolución geológica cenozoica del sur de México, y se plantean las principales interrogantes respecto a los diferentes episodios tectónicos y magmáticos que se han reconocido en la región. La revisión se enfoca principalmente en la Sierra Madre del Sur que, para los objetivos de este trabajo, se asume como la región montañosa limitada por la FVTM al norte, el Istmo de Tehuantepec y la Sierra de Juárez (terreno Juárez) al este, y la margen continental del Pacífico, al sur (Figura 1). La revisión no incluye la evolución de la FVTM porque se trata de un problema complejo que merece un tratamiento aparte. Tampoco se discute la evolución de las regiones de Chiapas, la llanura costera del Golfo de México ni la península de Yucatán, porque en su evolución intervinieron factores tectónicos del ámbito del Golfo de México y requieren también de un tratamiento aparte. Este trabajo no pretende ser exhaustivo en cuanto a compilar la mayor parte de los datos disponibles, pero si se intenta delinear las tendencias generales en las interpretaciones, además de comentar algunos de los datos y rasgos más relevantes. La información más importante sobre la edad y geoquímica de las rocas del sur de México puede ser consultada en artículos y bases de datos disponibles (i. e. Schaaf et al., 1995; Morán Zenteno et al., 1999; Garduño-Monroy et al., 1999; Ferrari et al., 1999 y la base de datos correspondiente; así como en la Western North America volcanic and intrusive rock database, http://navdat.geongrid.org/.

\section{Estructura cortical y entorno tectónico}

Con base en los contrastes petrológicos, tectónicos y geocronológicos de los segmentos de basamento premesozoico aflorantes, y tomando en cuenta los cambios en la naturaleza petrotectónica de las rocas mesozoicas que cubren y bordean el basamento antiguo, la corteza superior del sur de México ha sido subdividida en cinco terrenos tectonoestratigráficos: Oaxaca, Mixteca, Guerrero, Xolapa y Juárez (Campa y Coney, 1983) (Figura 1). Posteriormente, Sedlock et al. (1993) proponen una subdivisión similar con algunas variaciones en la posición de los contactos entre terrenos. El carácter tectónico y localización de los contactos entre estos terrenos han sido objeto de controversia (i. e., Sedlock et al., 1993; Centeno-García et al., 1993a; Lang et al., 1996; Freydier, et al., 1996; Elías Herrera et al., 2000; Cabral-Cano et al., 2000, Keppie, 2004); sin embargo, existe un consenso respecto a la existencia de contrastes litológicos a nivel de las rocas expuestos en superficie. Las interpretaciones sobre los contrastes corticales basados en datos geofísicos son escasas. Algunos modelos de la litósfera basados en perfiles magnetotelúricos han revelado que ciertos cambios laterales significativos en la conductividad eléctrica no siempre coinciden con los límites propuestos para los terrenos tectonoestratigráficos (Jording et al., 2000).

De manera simplificada se puede decir que en el sur de México los terrenos Guerrero y Juárez, que tienen una cobertura cretácica importante, delimitan con sus flancos oriente y poniente, respectivamente, a un núcleo del basamento premesozoico compuesto por los complejos metamórficos que conforman los terrenos Mixteco y Oaxaca, cuya cobertura cretácica es de espesor considerablemente menor. Los límites entre estos terrenos están caracterizados por estructuras con una orientación $\sim \mathrm{N}-\mathrm{S}$. Estas estructuras se encuentran truncadas hacia el sur por los afloramientos del terreno Xolapa que definen una franja paralela a la trinchera. En efecto, uno de los detalles más sobresalientes de la corteza del sur de México es el carácter truncado y exhumado de la margen continental del Pacífico (Bellon, et al., 1982; Morán Zenteno et al., 1996). La presencia abundante de rocas plutónicas del Cenozoico a lo largo de la margen, desde Manzanillo al Istmo de Tehuantepec, y del batolito del Cretácico Tardío de Puerto Vallarta, en la región de Jalisco (Figura 2), revela el levantamiento diferencial que ha tenido la margen continental con respecto a la zona interior, en donde se encuentran expuestas principalmente secuencias sedimentarias y volcánicas del Cretácico y Cenozoico. La distribución de rocas plutónicas de arco en la zona litoral, y aún en la zona marina cercana a la trinchera (Bellon et al., 1982), ponen de manifiesto, además de la exhumación de la margen continental, el avance que ha tenido la trinchera hacia el continente.

El truncamiento de la margen continental del Pacífico en el sur de México ha sido asociado de manera reiterada a la remoción del bloque de Chortis de la margen continental del sur de México (Malfait y Dinkelman, 1972; Ross y Scotese, 1988; Pindell et al., 1988, Schaaf et al., 1995) (Figura 3a). La migración del magmatismo de la Sierra Madre del Sur hacia el sureste y la presencia de zonas de cizalla con componente lateral izquierda han sido relacionadas con esta hipótesis (Herrmann et al., 1994; Schaaf et al., 1995; Morán- Zenteno et al., 1996). Sin embargo, el polo de rotación promedio estimado para la placa del Caribe con respecto a Norteamérica (Pindell et al., 1988) no es compatible con este desplazamiento si el bloque de Chortis hubiera sido parte de esta placa a partir del Eoceno-Oligoceno.

La tasa baja de acumulación de sedimentos en el prisma desarrollado en la trinchera confirma que existe una erosión por subducción activa en esta región (Ducea et al., 2004b). De acuerdo con los modelos basados en perfiles sísmicos a lo largo de la zona costera del oriente de Guerrero y Oaxaca, la corteza continental tiene entre 15 y $20 \mathrm{~km}$ de espesor (Nava et al., 1988). Los modelos sobre la geometría de la zona de subducción indican una amplia zona de contacto de la base de la corteza con el segmento subducido de la placa de Cocos por lo menos hasta distancias de $100 \mathrm{~km}$ de la costa (Valdés, et al., 1986; GEOLIMEX working group, 1994; Pardo y Suárez, 1995). Estas interpretaciones 

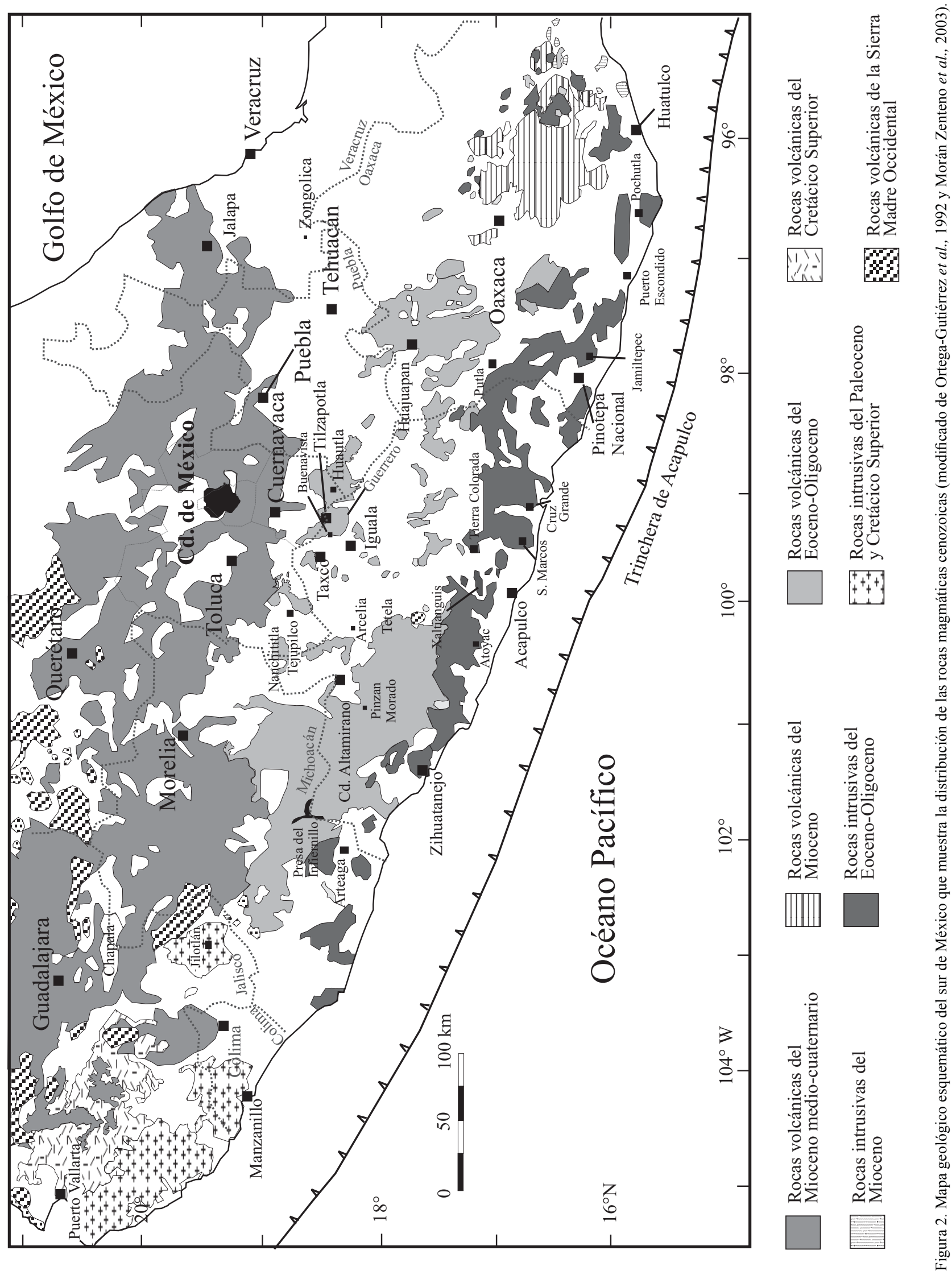
implican que ocurrieron cambios significativos en la geometría de la subducción posteriores al desarrollo del arco magmático representado por los plutones terciarios de la margen continental.

La disminución en el ángulo de subducción y el desplazamiento de la trinchera hacia el continente, también implican la remoción de un segmento de la cuña del manto y la parte inferior de la corteza continental en la zona de la margen continental actual. De acuerdo con el modelado gravimétrico de Urrutia-Fucugauchi y Flores Ruiz (1996) y el modelado sísmico de Valdés et al. (1986), el espesor de la corteza continental, de la costa al sector este de la Faja Volcánica Transmexicana, varía de 20 a $45 \mathrm{~km}$, mientras que en la parte oriental de la Faja Volcánica Transmexicana alcanza hasta $50 \mathrm{~km}$.

En la actualidad, la litósfera oceánica que subduce a lo largo de la trinchera de Acapulco está dividida en los segmentos correspondientes a la placa de Cocos y a la placa de Rivera (Figura 1). La placa de Cocos tiene un desplazamiento relativo con respecto a la zona continental de México definido por un polo de rotación ubicado frente a las costas de Baja California. Su velocidad de convergencia varía de NW a SE de 5.5 a $6.8 \mathrm{~cm} /$ año (Pardo y Suárez 1995; DeMets y Traylen, 2000). La placa de Rivera subduce a la placa de Norteamérica a lo largo del segmento de trinchera correspondiente a la zona de Jalisco y según un polo de rotación ubicado cerca de la boca del Golfo de California (DeMets y Stein, 1990; DeMets y Wilson; 1997). La velocidad de convergencia de la placa de Rivera frente a la costa de Manzanillo ha sido calculada en 3.8 $\mathrm{cm} /$ año (DeMetz y Traylen, 2000). A lo largo de la trinchera Mesoamericana en el sur de México inciden las zonas de fractura Rivera, Orozco y O'Gorman (Figura 1), la primera de la cuales representa el límite entre las placas de Rivera y Cocos y cuya subducción ha inducido extensión en la zona continental (Ferrari et al., 1994; Bandy et al., 1995). Para las zonas continentales ubicadas frente a las fracturas de Orozco y O'Gorman no se han reconocido hasta ahora rasgos geológicos que resultaran de su intersección con la trinchera. En la zona del Istmo de Tehuantepec incide en forma oblicua la prominencia lineal correspondiente al a la zona de fractura de Tehuantepec. El punto en la que esta fractura intercepta a la trinchera coincide aproximadamente con lo que ha sido considerado la unión triple difusa de las placas del Caribe, Norteamérica y Cocos, así como con un cambio notable en el ángulo de subducción $\left(10^{\circ}\right.$ al oeste de este punto y $45^{\circ}$ al este) (Pardo y Suárez, 1995). Debajo del Istmo de Tehuantepec se define una zona lineal con sismicidad frecuente que es perpendicular a la trinchera (Ponce et al., 1992). Las fallas cenozoicas de desplazamiento principalmente izquierdo reconocidas en el sureste de Oaxaca y en Chiapas, han sido principalmente relacionadas a la evolución reciente del punto triple trinchera-trincheratransforme (Delgado Argote y Carballido Sánchez, 1990; Tolson, 1998; Guzmán Speziale y Meneses Rocha, 2000). Barrier et al. (1998) han reconocido, por otra parte, una
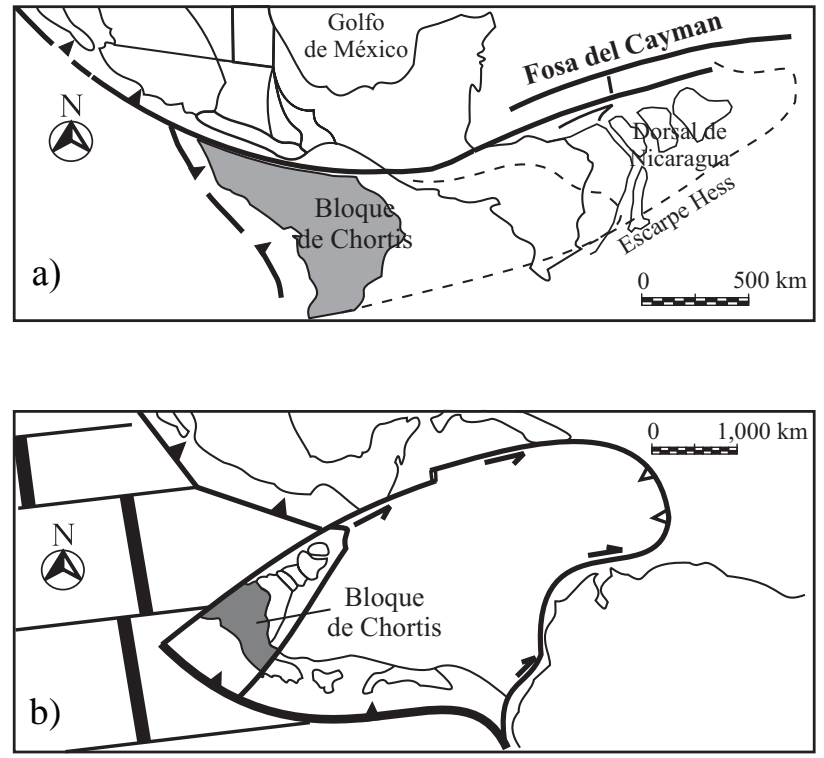

Figura 3. Reconstrucciones tectónicas para el Eoceno que muestran las posiciones alternativas que se han sugerido para el bloque Chortis (en gris). La figura (a) corresponde a la reconstrucción de Schaaf et al. (1995), modificada de la reconstrucción previa de Ross y Scotese (1988), y basada en los patrones de extinción magmática documentados con fechamientos isotópicos. La figura (b) corresponde a la reconstrucción alternativa de Keppie y Morán-Zenteno (2004) basada en un polo de rotación promedio del Eoceno ubicado cerca de Santiago de Chile (Pindell et al., 1988), definido por la traza general del límite entre las placas del Caribe y Norteamérica. La terminación occidental del sistema de fallas PolochicMotagua con concavidad al norte es diferente a la fosa del Cayman y puede deberse a la interacción transpresiva reciente (Neógeno) del bloque de Chortis con el segmento chiapaneco del bloque de Yucatán.

zona de falla normal del Mioceno-Plioceno con dirección $\mathrm{N}-\mathrm{S}$ en el Istmo de Tehuantepec que han atribuido a la influencia de la subducción de la fractura de Tehuantepec y los cambios en la geometría de la placa subducida. Estos autores atribuyen, además, la extensión $\mathrm{N}-\mathrm{S}$ más reciente en el Golfo de Tehuantepec a la influencia del movimiento hacia el este de la placa del Caribe.

De acuerdo a las interpretaciones basadas en la edad y configuración de las anomalías magnéticas del piso oceánico, la cinemática de los segmentos de litósfera oceánica subducida a lo largo de la trinchera ha sufrido transformaciones relacionadas con saltos de diferentes segmentos de la Dorsal del Pacífico del Este en episodios que ocurrieron hace 25, 12.5-11 y 6.5-3.5 Ma (Mammerickx y Klitgor, 1982). Estos episodios se asociaron a cambios en los polos de rotación, pero también implicaron la segmentación de la antigua placa de Farallón en las placas de Cocos y Rivera. No se tienen evidencias firmes de que los cambios en la cinemática de los segmentos de litósfera oceánica del Pacífico hayan sido los causantes de los cambios en la geometría de la subducción, pero la simultaneidad aparente en estos cambios y las variaciones introducidas en la velocidad de convergencia, sugieren una relación causa-efecto. 


\section{Deformación orogénica de fines del Cretácico y establecimiento del dominio continental}

Hacia el Cretácico Tardío, un evento mayor de deformación progresiva por acortamiento, con dirección de transporte predominante hacia el este-noreste provocó el plegamiento y cabalgamiento de las secuencias marinas del Mesozoico y anteriores. Este evento ha sido relacionado con la orogenia Laramide del suroeste de la cordillera norteamericana por su edad y estilo similares (Campa et al., 1976; Campa y Ramírez, 1979; Salinas-Prieto et al., 2000; Dickinson et al., 2001). En la Sierra Madre del Sur, las evidencias del acortamiento se presentan en una amplia zona entre los estados de Michoacán y Veracruz, aunque es en el estado de Guerrero donde ha sido estudiado recientemente con mayor detalle (Lang et al., 1996; Cabral-Cano et al., 2000a; Salinas-Prieto et al., 2000; Elías-Herrera et al., 2000).

El estilo estructural en el sur de México consiste en pliegues y cabalgaduras de bajo ángulo con orientación aproximada norte-sur y dirección predominante de transporte tectónico hacia el oriente, con fallas asociadas de desplazamiento lateral. También se ha documentado una vergencia opuesta en la zona de Teloloapan, interpretada como resultado del acortamiento progresivo (Salinas-Prieto et al., 2000), y en la zona de la Plataforma GuerreroMorelos, interpretada como resultado del contraste lateral de resistencia entre las secuencias marinas mesozoicas y las rocas metamórficas del Complejo Acatlán (Cerca et al., 2004).

Las diferencias en el estilo estructural asociadas con cambios litológicos y de topografía permiten dividir los afloramientos con estructuras de acortamiento de la Sierra Madre del Sur en tres zonas (Figura 4). La zona occidental incluye las estructuras de bajo ángulo y orientación nortesur, que afloran desde la zona de Arcelia hasta la parte media de la Plataforma Guerrero Morelos, al oriente de la cabalgadura de Teloloapan. Las estructuras principales en esta zona involucran la deformación de rocas de la cobertura cretácica. En algunas zonas específicas como en Tejupilco, Estado de México (Elías-Herrera et al., 2000), Pinzón Morado, Guerrero (Consejo de Recursos Minerales, 2000), y Arteaga, Michoacán (Centeno-García et al., 1993b), se encuentran expuestas rocas sedimentarias metamórficas de edad precretácica que conforman altos estructurales del basamento. Cabe señalar que la zona al poniente de Arcelia se encuentra cubierta por extensos depósitos continentales que aparentemente varían en edad desde el Cretácico más tardío hasta el Paleógeno (Jansma y Lang, 1997). En la región de Tiquicheo, Benammi, et al. (2005) han reportado recientemente el hallazgo de restos de dinosaurios del Cretácico Tardío en capas continentales que cubren en discordancia a secuencias plegadas del terreno Guerrero, lo que indica que la deformación orogénica principal culminó antes del final del Cretácico en esa región.

En contraste, la zona central se encuentra asociada a extensos afloramientos de un núcleo metamórfico premesozoico (Figura 4). Incluye parcialmente las estructuras en la zona oriental de la Plataforma Guerrero-Morelos y los afloramientos de rocas metamórficas al oriente de la falla Oaxaca. En esta zona se observan las perturbaciones más evidentes del patrón laramídico de orientación norte-sur observado al poniente, provocadas probablemente por desplazamientos verticales y laterales del núcleo metamórfico durante una fase de deformación en el Paleógeno (Cerca et al., 2004). De acuerdo a este autor, el fuerte contraste mecánico entre el núcleo metamórfico y las secuencias sedimentarias adyacentes controló la concentración de la deformación en los bordes del bloque más competente. De esta manera, la geometría de las estructuras de acortamiento en esta zona está definida por pliegues y cabalgaduras que definen una saliente en forma de arco convexo hacia el norte, con vergencia radial hacia el exterior del bloque y la deformación dentro del bloque es menos intensa.

Por ultimo, la zona oriental comprende las estructuras entre la Sierra de Juárez, Sierra de Zongolica y la cabalgadura de Vista Hermosa (Figura 4). El estilo estructural predominante en esta zona consiste de un cinturón de pliegues y cabalgaduras con una orientación general noroestesureste y una vergencia principal hacia el noreste. En esta zona se ha interpretado que la fase tectónica es continua hasta el Eoceno (Meneses-Rocha et al., 1996; Eguiluz de Antuñano et al., 2000).

La edad de la deformación laramídica ha sido delimitada en la cordillera norteamericana entre 75 y $35 \mathrm{Ma}$ (Dickinson et al., 1988; Bird, 1998) con un máximo de deformación en el Eoceno en el frente orogénico de la Sierra Madre Oriental (Eguiluz de Antuñano et al., 2000 y referencias citadas en ese trabajo). En el sur de México, se ha interpretado que el acortamiento comienza con el ahogamiento de las plataformas de carbonatos y el inicio de la sedimentación clástica que se ha ubicado en la frontera Cenomaniano-Turoniano (ca.93 Ma) (Hernández-Romano et al., 1997; Aguilera-Franco y Hernández-Romano, 2004) o en la frontera Turoniano-Coniaciano (ca. $89 \mathrm{Ma}$ ) (Lang y Frerichs, 1998). Esta edad es consistente con las edades mas jóvenes de la secuencia volcánica submarina plegada del Cretácico que alcanzan entre 93 y 88 Ma en la zona de Teloloapan y Arcelia (Delgado-Argote et al., 1992; ElíasHerrera et al., 2000; Cabral-Cano et al., 2000a).

Las edades reportadas de finalización de la orogenia Laramide en el sur de México parecen migrar de poniente a oriente. En la zona occidental del sur de México hay edades de magmatismo considerado postorogénico desde el final del Maastrichtiano en la zona occidental (OrtegaGutiérrez, 1980; González-Partida et al., 2003; Levresse et al., 2004; Cerca et al., 2004). También dentro de esta zona hay algunas estructuras como el pliegue de Tzitzio, cuya cinemática y edad de deformación no están bien comprendidas y parecen constituir una anomalía con respecto al patrón de deformación progresiva por acortamiento en la región. Los datos palinológicos sugieren que las 


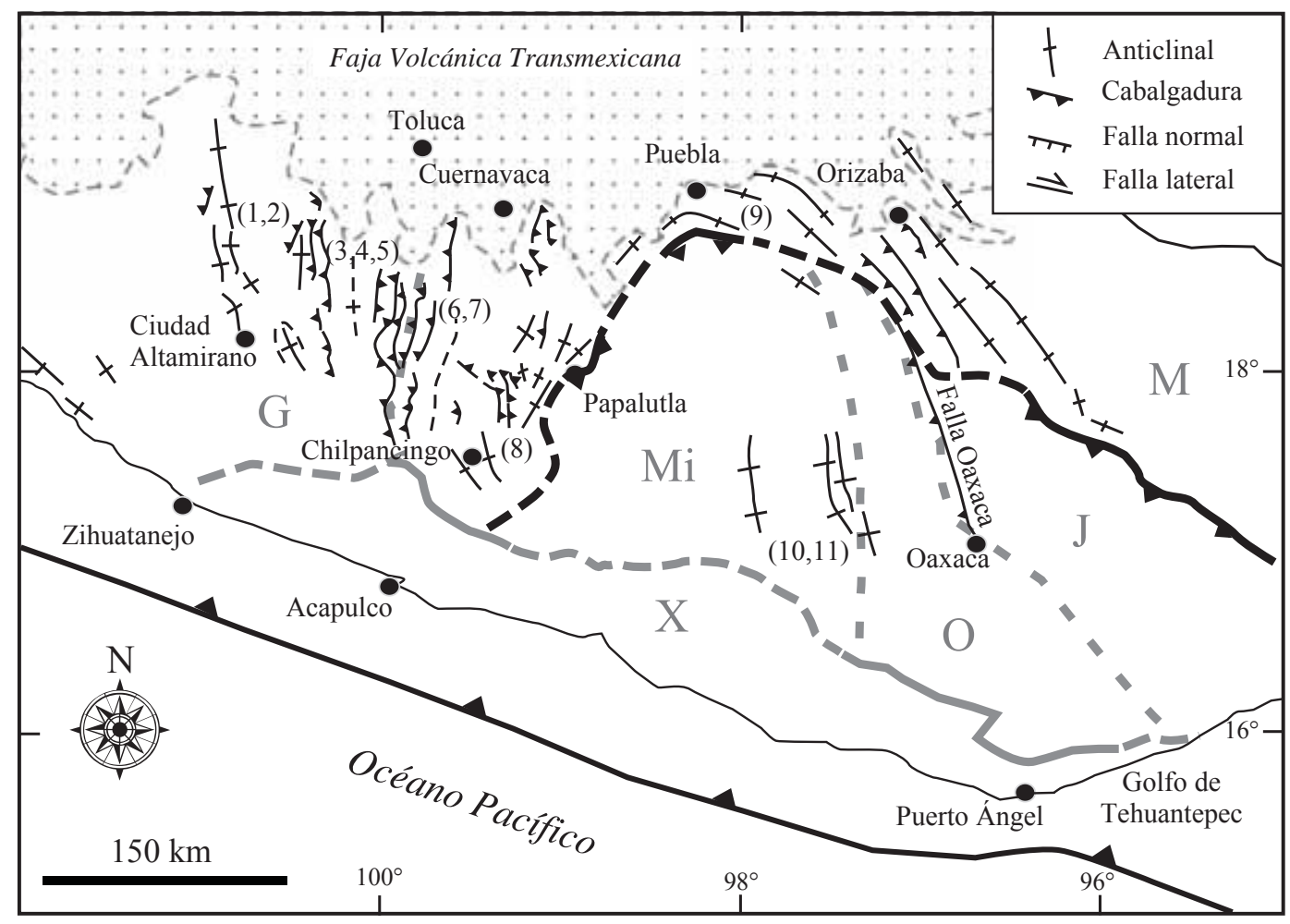

Figura 4. Esquema que muestra las estructuras de acortamiento documentadas que afectan a las rocas del Cretácico Superior y Paleógeno en el sur de México (Cerca et al., 2004). Las líneas punteadas de color gris indican las fronteras de los terrenos tectonoestratigráficos modificadas a partir de Campa y Coney (1983) y Sedlock et al. (1993). Los terrenos se indican con las letras mayúsculas: G, Guerrero; Mi, Mixteco; O, Oaxaca; J, Juárez; M, Maya; y X, Xolapa. Las líneas punteadas de color negro indican los límtes aproximados de afloramientos de rocas premesozoicas, mientras que las líneas discontinuas indican las zonas donde rocas paleozoicas cabalgan sobre rocas mesozoicas. Las estructuras fueron compiladas de: 1) Montiel-Escobar et al. (2000); 2) Altamira-Areyán (2002); 3) Elías-Herrera et al. (2000); 4) Salinas-Prieto et al. (2000); 5) Cabral-Cano et al. (2000b); 6) Rivera-Carranza et al. (1997); 7) Campa-Uranga et al. (1998); 8) Cerca et al. (2004); 9) Monroy y Sosa (1984); 10) Meneses-Rocha et al. (1994); 11) González-Ramos et al. (2001); y referencias incluidas en esos trabajos.

secuencias continentales involucradas en la deformación por acortamiento de la zona de Tzitzio alcanzan una edad paleocénica (Altamira-Areyan, 2002). Por otro lado, en la zona de Zongolica y aún más al oriente, se ha propuesto que la deformación llega hasta el Eoceno tardío (Mossman y Viniegra, 1976; Eguiluz de Antuñano et al., 2000). A nivel general, durante y hacia el final del proceso de deformación, el medio ambiente de depósito cambió en la Sierra Madre del Sur progresivamente de marino a continental. El establecimiento del dominio continental migró de poniente a oriente y los nuevos productos volcánicos y sedimentos fueron depositados en cuencas intermontanas (Figuras 5 y 6).

\section{Magmatismo y sedimentación continental del Maastrichtiano-Paleoceno}

La información geocronológica reciente revela que durante el Maastrichtiano y Paleoceno, el magmatismo tenía una distribución más amplia de lo que se había supuesto. Hasta ahora se pueden delinear dos zonas principales de actividad ígnea de arco. Por una parte, existen extensos cuerpos plutónicos y algunas rocas volcánicas de este intervalo en una amplia región en Jalisco, Colima y Michoacán (Schaaf et al., 1995; Moran-Zenteno et al., 1999 y referencias incluidas). Por otra parte, se han reconocido intrusivos y rocas volcánicas de menor volumen en la región de Guerrero y Estado de México ubicada entre los meridianos $99^{\circ}$ y $100^{\circ}$ (Figura 7a).

En la porción occidental de la Sierra Madre del Sur, se han documentado secuencias de rocas que registran el intervalo de tiempo entre el Maastrichtiano y el Eoceno tardío, y consisten principalmente de depósitos sedimentarios de ambiente continental, con rocas volcánicas intercaladas $(e . g$., Grupo Balsas, Formación Tetelcingo) (Fries, 1960; Ortega Gutiérrez, 1980) (Figuras 5 y 6). El espesor considerable de estas secuencias sugiere que rellenaron cuencas continentales. Estas cuencas se encontraban limitadas por pliegues y cabalgaduras formadas durante la deformación laramídica y por fallas transcurrentes y normales contemporáneas a su depósito (Figuras 5 y 6 ).

Desde las primeras descripciones del Grupo Balsas se consideró que algunas de las rocas sedimentarias en la base podrían incluir rocas del Cretácico Superior (Fries, 1960). 


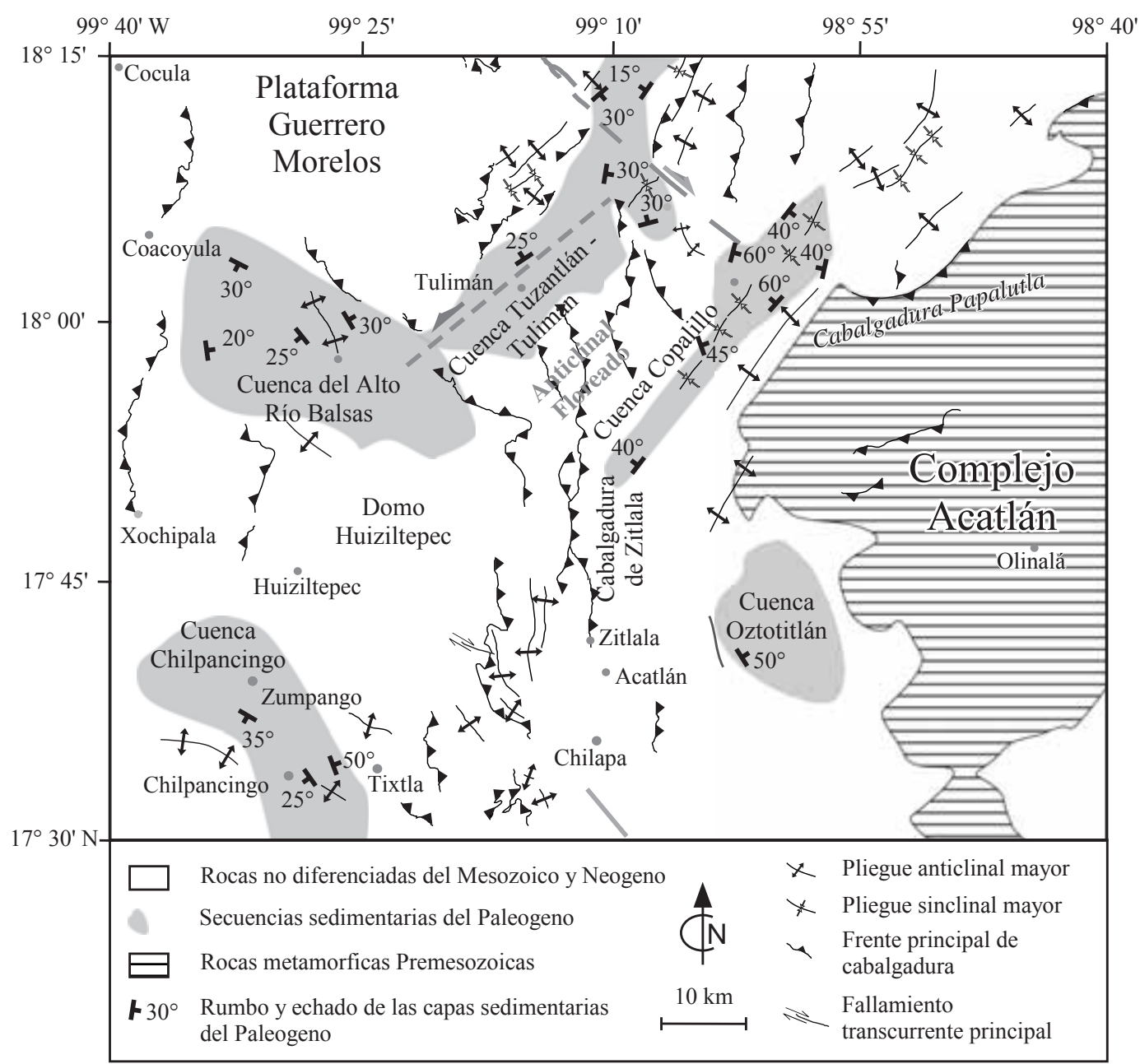

Figura 5. Esquema estructural simplificado de la zona de la Plataforma Guerrero Morelos, adyacente al flanco poniente del terreno Mixteco. En esta figura se muestra la distribución aproximada de algunas cuencas (en gris) con registro sedimentario del Paleógeno. Estas cuencas se encuentran delimitadas por estructuras de acortamiento y son afectadas por fallas con desplazamiento lateral (modificado de Cerca et al., 2004).

Las edades de rocas volcánicas e intrusivas entre 67 y $\sim 55$ Ma, distribuidas desde la zona de Mezcala hasta la región de Acapulco (Ortega-Gutiérrez, 1980; Morán-Zenteno, 1992; Meza- Figueroa et al., 2003; González-Partida et al., 2003; Tritlla et al., 2003; Ducea et al., 2004a), sugieren que un episodio magmático regional ocurrió al finalizar el acortamiento Laramide en esta región (Figura 7a).

En la zona de Mezcala (Figura 7a) las rocas intrusivas se encuentran emplazadas en lineamientos orientados noroeste-sureste, las cuales cortan a las cabalgaduras, y han arrojado edades de enfriamiento $\mathrm{Ar}-\mathrm{Ar}$ (biotita) y de $\mathrm{U}-\mathrm{Pb}$ (circones) entre 68 y $60 \mathrm{Ma}$ (Meza-Figueroa et al., 2003; González-Partida et al., 2003;). Algunas de las peculiaridades mineralógicas y químicas de estas rocas se han interpretado como características de intrusivos adakíticos, producto de la fusión parcial de la placa subducida en la transición anfibolita-eclogita que reaccionó con una cuña de manto al terminar la subducción horizontal (GonzálezPartida et al., 2003). Según Meza-Figueroa et al. (2003), la diferencia en las características geoquímicas con respecto a los plutones del Terciario medio indica también diferentes orígenes petrogenéticos. De edad similar a estos intrusivos, en la zona del Alto Río Balsas, se encuentra una secuencia volcánica y sedimentaria llamada Formación Tetelcingo. Ortega-Gutiérrez (1980) dividió esta secuencia volcánica en tres partes sucesivas, brechas y tobas, lavas y brechas, y brechas piroclásticas. Las lavas varían de composición entre andesítica y riolítica (De Cserna et al., 1980). Entre las lavas hay estratos potentes de brechas volcánicas y sedimentos de ambiente lacustre-fluvial, principalmente conglomerados. Las edades $\mathrm{K}-\mathrm{Ar}$ para la base y en la mitad de la secuencia son $68.8 \pm 2.4$ y $66 \pm 2.3 \mathrm{Ma}$, respectivamente (Ortega-Gutiérrez, 1980).

Edades similares o cercanas se han documentado en Peña Colorada, Colima (Tritlla et al., 2003 y referencias incluidas), en rocas volcánicas al norte de Zihuatanejo, Guerrero (Garduño-Monroy et al., 1999), en intrusivos extensos en el bloque Jalisco (Schaaf et al., 1995), y en algunos plutones de la región de Acapulco (Morán-Zenteno et al., 1992; Ducea et al., 2004a) (Figura 7a). 


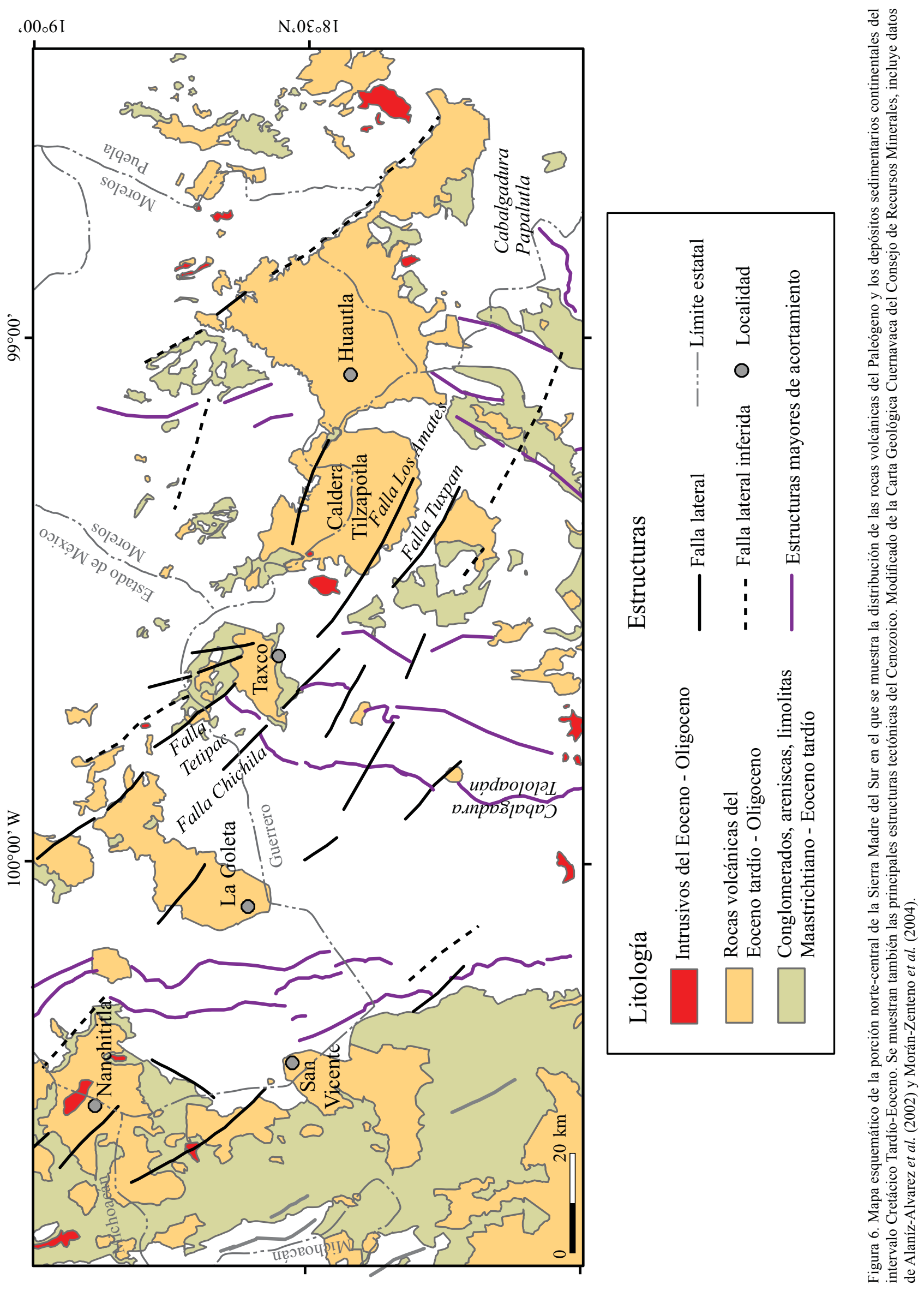


Aunque la geometría y el volumen de los productos de este episodio magmático son desconocidos, y las exposiciones se encuentran en áreas relativamente restringidas, la distribución geográfica amplia de las muestras sugiere que fue un evento de escala regional. En la zona de Mezcala, los intrusivos se encuentran relacionados aparentemente con estructuras con orientación noroeste-sureste (González-Partida et al., 2003), oblicuas con respecto a las estructuras de acortamiento. Se ha documentado que las estructuras eocénicas con esta orientación, en la parte norte de la Sierra Madre del Sur, son consistentes con un régimen de desplazamiento lateral (Morán-Zenteno et al., 1999; Alaniz-Álvarez et al., 2002). Si las rocas volcánicas del Maastrichtiano-Paleoceno también se emplazaron en estructuras localizadas dentro de un régimen de desplazamiento lateral, esto podría tener implicaciones tectónicas importantes. Se puede especular que un aumento en el ángulo de subducción de la placa de Farallón haya ocurrido al final del acortamiento laramídico (MaastrichtianoPaleoceno). Las diferencias de ángulo y velocidad entre segmentos de la placa pudieron generar discontinuidades perpendiculares a la trinchera, donde se emplazaron cuerpos magmáticos (Cerca et al., 2004). Sin embargo, un aumento en el ángulo de subducción no explica satisfactoriamente la migración hacia el sureste en la edad de los plutones a lo largo de la costa desde el Cretácico Tardío, ni la presencia de dos zonas magmáticas de edad similar (PaleocenoEoceno temprano), ubicadas a distancias diferentes de una eventual paleotrinchera con orientación norte-sur (Figura 7a). La presencia de estas dos zonas magmáticas del Paleoceno-Eoceno temprano puede ser más compatible con una paleotrinchera paralela a la trinchera actual, lo que pone en duda la presencia del bloque de Chortis para este tiempo frente a la margen continental del sur de México. Sin embargo, esta alternativa no permite explicar con claridad la migración de la edad del magmatismo a lo largo de la costa, ni la orientación preferencial al norte de los pliegues y las cabalgaduras laramídicas.

\section{Plutonismo y evolución de la margen continental}

La región de la margen continental del sur de México cuenta con afloramientos extensos de cuerpos plutónicos del Cretácico más tardío y del Terciario que frecuentemente alcanzan dimensiones batolíticas (Figura 2). Aproximadamente el 50\% de la franja continental de 100 $\mathrm{km}$ de ancho, a partir de la costa, está ocupada por afloramientos de estos cuerpos que en conjunto cubren un área aproximada de $30000 \mathrm{~km}^{2}$. En los sondeos realizados en la zona marina ubicada entre la costa y la trinchera (crucero 66 del Deep Sea Drilling Project) (Bellon et al., 1982) y en los dragados frente a las costas de Jalisco (Mercier de Lépinay et al., 1997) se han reportado también cuerpos plutónicos que indican su proximidad inmediata a la trinchera. Existen también cuerpos deformados de origen plutónico que han sido atribuidos al Complejo Xolapa (Ortega-Gutiérrez, 1981) y cuya edad ha sido materia de controversia (Robinson, 1989; Morán-Zenteno, 1992; Herrmann et al., 1994; Ducea et al., 2004a).

La composición de los cuerpos plutónicos de la margen continental es dominantemente silícica y subalcalina, con una representación mayoritaria de composiciones granodioríticas y tonalíticas, y volúmenes subordinados de granito (Schaaf et al., 1995; Morán-Zenteno et al., 1999). Los principales minerales ferromagnesianos dominantes en estas rocas son la hornblenda y la biotita. Existen también volúmenes subordinados de intrusivos de composición gabroica y diorítica, especialmente en el sector de Manzanillo (Figura 2) (Schaaf, 1990).

Las relaciones isotópicas iniciales de ${ }^{87} \mathrm{Sr} /{ }^{86} \mathrm{Sr},{ }^{143} \mathrm{Nd} /$ ${ }^{144} \mathrm{Nd}$ y de $\mathrm{Pb}$ de las rocas magmáticas del sur de México, presentan una variación relativamente baja. En el caso del ${ }^{87} \mathrm{Sr} /{ }^{86} \mathrm{Sr}$ inicial las rocas magmáticas cenozoicas varían típicamente de 0.7035 a 0.7055 (Figura 8), con el mayor número de valores entre 0.7039 y 0.7046 , mientras que los valores iniciales de $\varepsilon N d$ varían típicamente de $+0.5 \mathrm{a}+5.7$ con la mayor parte de los valores entre +1 y +3 (MoránZenteno et al., 1999 y referencias incluidas). Valores extremos de hasta +5 se alcanzan en los intrusivos cenozoicos ubicados entre Zihuatanejo y Manzanillo, pero también en rocas volcánicas de la zona de Tilzapotla en el estado de Morelos (Figura 2) (Schaaf, 1990; Morán-Zenteno et $a l ., 2004)$. Valores negativos de hasta -3 se observan en los plutones silícicos de la región de Puerto Escondido (Hernández-Bernal y Morán-Zenteno, 1996). El batolito cretácico de Puerto Vallarta presenta, en contraste, valores más negativos de $\varepsilon N d(\sim-7)$ y valores más altos de ${ }^{87} \mathrm{Sr} /{ }^{86} \mathrm{Sr}$ (Schaaf, 1990). En el caso de las firmas isotópicas de $\mathrm{Pb}$ las rocas magmáticas cenozoicas del sur de México varían típicamente dentro de los rangos ${ }^{206} \mathrm{~Pb} /{ }^{204} \mathrm{~Pb}=18.65-18.97$, ${ }^{207} \mathrm{~Pb} /{ }^{204} \mathrm{~Pb}=15.55-15.65,{ }^{208} \mathrm{~Pb} /{ }^{204} \mathrm{~Pb}=38.25$ a 38.82 (Figura 9) (Herrmann et al., 1994; Martiny et al., 2000; Martiny et al., 2002; Morán-Zenteno et al., 2004). Los datos isotópicos de Sr no muestran diferencias considerables con respecto a los valores reportados de la Sierra Madre Occidental; sin embargo, en el caso de los datos isotópicos de $\mathrm{Pb}$ las rocas magmáticas del sur de México indican una fuente de contaminación cortical más homogénea con respecto a la que experimentaron las rocas de la Sierra Madre Occidental. En general, las rocas graníticas cenozoicas de la margen del Pacífico del sur de México se pueden considerar como granitos de tipo I o granitos de hornblenda, en el sentido de Chapell y White (1974), y de Kemp y Hawkesworth (2004)

La variabilidad restringida de las firmas isotópicas y su tendencia hacia valores relativamente primitivos de las rocas magmáticas cenozoicas indican una relativamente baja contaminación por componentes de la corteza antigua, comparada con otros sectores de la Cordillera Norteamericana. La composición dominantemente silícica y el gran volumen de los plutones distribuidos en la margen continental sugie- 

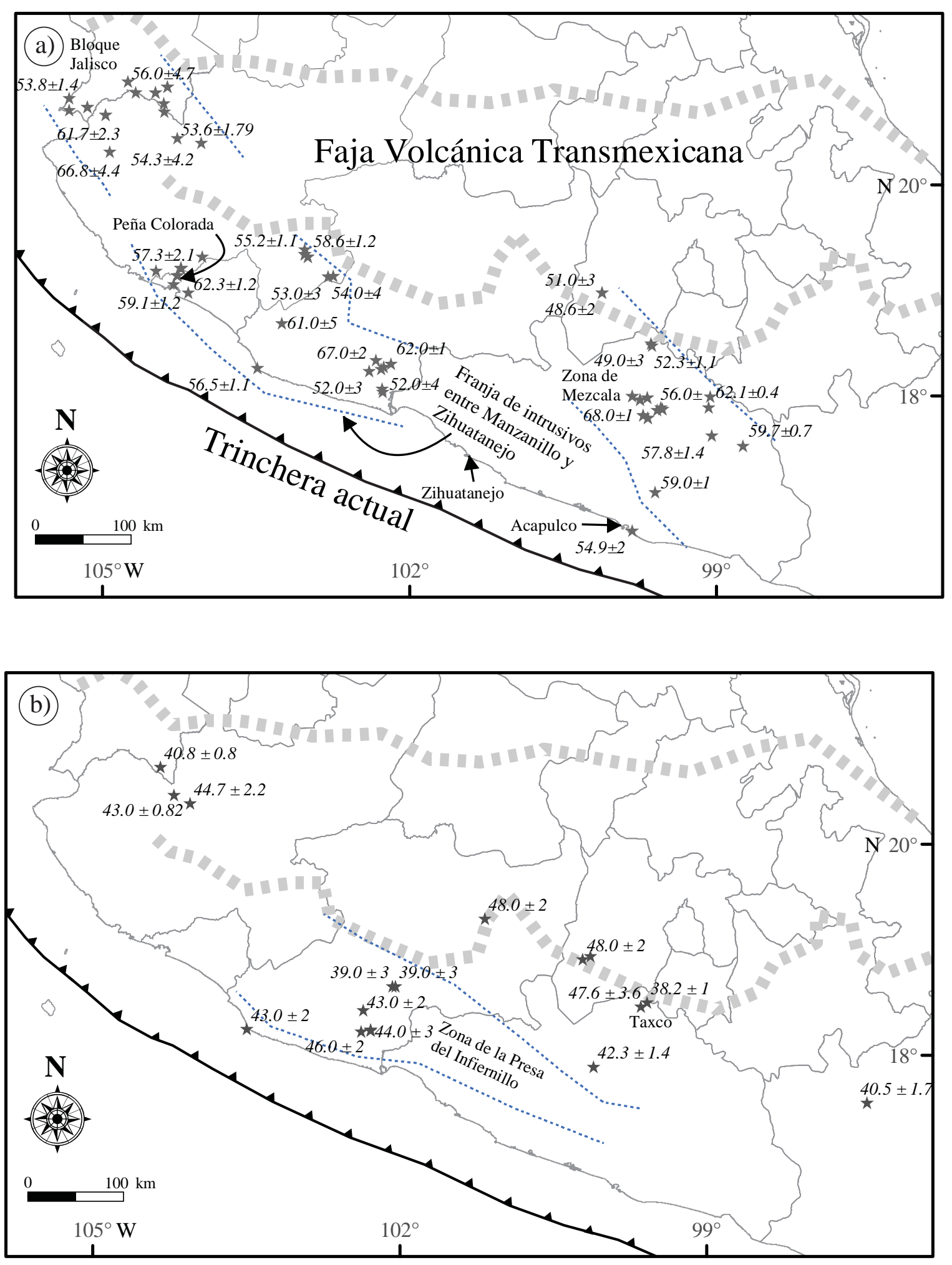

Figura 7. Distribución espacio-temporal de las rocas magmáticas terciarias del sur de México. Compilación de edades agrupadas en intervalos de (a) 68 a 48, (b) 48 a 38, y (c) 38 a 28 millones de años. Las edades disponibles han sido obtenidas mediante diversos métodos (K-Ar, Ar-Ar, Rb-Sr, U-Pb) los cuales se interpretan y tienen limitaciones diferentes. A pesar de estas limitaciones, el análisis de todo el conjunto permite delinear algunos patrones generales de la distribución de los eventos térmicos-magmáticos en la zona durante el Cenozoico. Se muestran algunos ejemplos de los datos en las etiquetas de las edades. Datos tomados de Schaaf et al. (1995); Morán-Zenteno et al. (1999); Ferrari et al. (1999); Garduño-Monroy et al., 1999; Cerca (2004); the western North America volcanic and intrusive rock database; y de los trabajos de Meza-Figueroa et al. (2003); Ducea et al. (2004); MoránZenteno et al. (2004) y Levresse et al. (2004). 


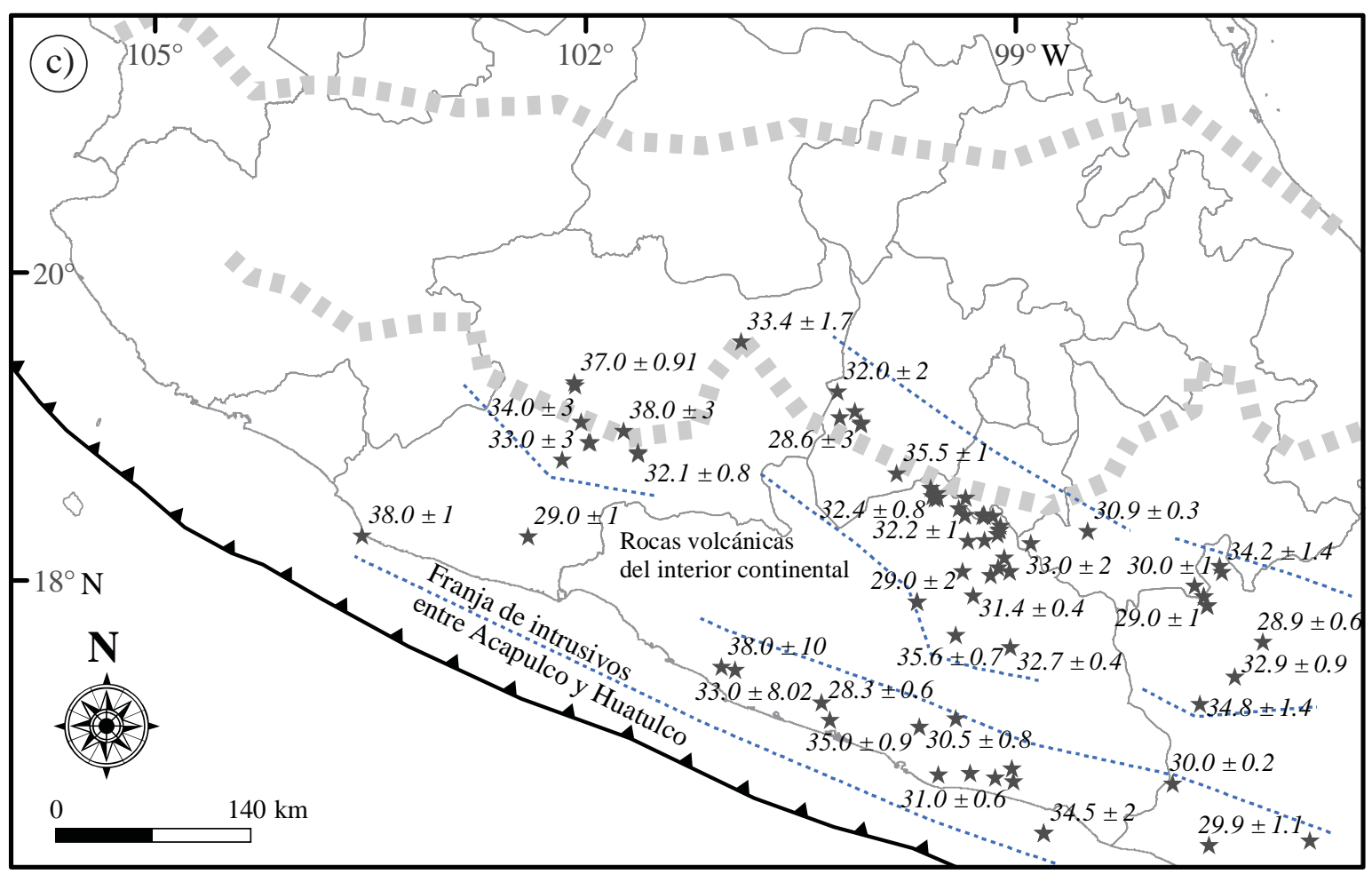

Figura 7. Continuación.

re que la fuente de los magmas debió ser una capa máfica recientemente adherida a la base de la corteza y alterada por metasomatismo. Debido a la imposibilidad petrogenética de generar grandes volúmenes de rocas plutónicas silícicas directamente de la cuña del manto, recientemente se ha interpretado este tipo de plutonismo en términos de un proceso en dos etapas (Pitcher, 1993) o que puede ser continuo (Kemp y Hawkesworth, 2004) pero que involucre, (1) la formación de una capa de composición basáltica en la base de la corteza (underplating) o la hibridización de la base de la corteza continental por la intrusión de magmas máficos provenientes del manto $\mathrm{y}$, (2) la posterior fusión parcial de la base máfica metasomatizada o híbridizada de la corteza por el nuevo arribo de magmas máficos, y la mezcla de estos con los productos de la fusión parcial de la base de la corteza. En todo caso, se tienen evidencias de que el gran pulso magmático silícico del Eoceno-Oligoceno en el sector Acapulco-Huatulco estuvo asociado a la formación $\mathrm{y}$ ascenso de magmas máficos. El pulso de magmatismo máfico proveniente del manto es necesario para que exista una transferencia de calor efectiva, capaz de producir la fusión parcial de la corteza basal máfica o híbrida producida durante eventos anteriores de adición de magmas máficos. La presencia de enjambres de diques con composición intermedia y máfica que cortan los plutones silícicos en la región de Tierra Colorada, Guerrero, sugiere esta relación. En la margen de los diques suele presentarse foliación cristal-plástica, lo que sugiere que los plutones todavía conservaban parte de su calor de emplazamiento.
La edad de cristalización de los plutones a lo largo de la margen varía desde $100 \mathrm{Ma}$ (Albiano), en la región de Puerto Vallarta, hasta $27 \mathrm{Ma}$ en la región de Huatulco, con los plutones cenozoicos $(<65 \mathrm{Ma})$ distribuidos principalmente al sureste de Manzanillo (Schaaf et al. 1995 y referencias incluidas) (Figura 10). A partir de las edades disponibles se ha sugerido que existe una migración del magmatismo hacia el sureste (Schaaf et al., 1995). La migración fue aparentemente más lenta en el sector Puerto Vallarta-Zihuatanejo (100-40 Ma) que en el de AcapulcoHuatulco (34-29Ma), con excepción del intrusivo de Acapulco y otros plutones deformados), en donde ocurrió en un lapso relativamente corto (Herrmann et al., 1994). Con base en fechamientos de $\mathrm{U}-\mathrm{Pb}$ realizados en cristales individuales de circones en este último sector, Ducea $e t$ al. (2004a) proponen que la migración del magmatismo no es evidente y que su extinción parece haber sido simultánea. Sin embargo, aunque sus edades de rocas plutónicas cenozoicas se restringen a una parte limitada de la margen en el sector de Acapulco-Puerto Escondido, en los datos geocronológicos se observa una pequeña, pero significativa diferencia, con una tendencia decreciente al sureste. Las edades de enfriamiento obtenidas por medio de fechamientos de $\mathrm{K}-\mathrm{Ar}$ y $\mathrm{Rb}-\mathrm{Sr}$ también expresan esta migración a lo largo de toda la margen continental de noroeste a sureste (Schaaf et al., 1995) (Figura 10). El intrusivo de Acapulco constituye una anomalía en el patrón general de migración, ya que se ha obtenido una edad de $\mathrm{U}-\mathrm{Pb}$ en circones de $54.9 \pm 2 \mathrm{Ma}$ (Ducea et al., 2004a) y 


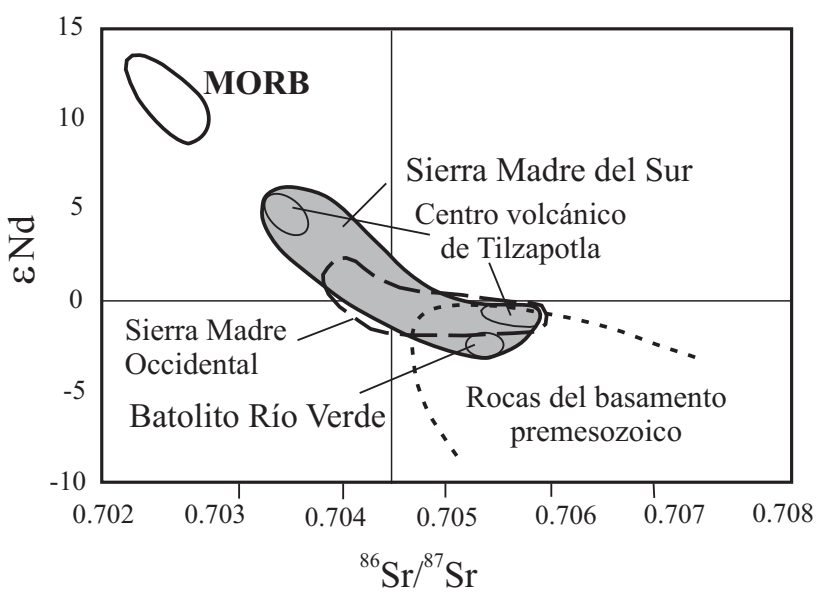

Figura 8. Distribución de las relaciones isotópicas iniciales de las rocas magmáticas del sur de México (SMS) en un diagrama de ${ }^{87} \mathrm{Sr}^{86} \mathrm{Sr}_{(i)}$ vs. $\varepsilon_{(\mathrm{Ni})}$. Como referencia se muestra el campo de distribución típico de las rocas volcánicas de la Sierra Madre Occidental (SMO) y algunas zonas del sur de México que presentan valores extremos. Los datos fueron tomados de Schaaf (1990), Schaaf et al. (1995 y referencias incluidas), Morán-Zenteno et al. (1999, 2004 y referencias incluidas), Martiny et al. (2000 y referencias incluidas).

edades de enfriamiento de $\mathrm{Rb}-\mathrm{Sr}$ y $\mathrm{K}$-Ar que varían de 43 a $50 \mathrm{Ma}$ (López Infanzón y Grajales Nishimura, 1984; Guerrero-García, 1975; Schaaf et al., 1995). Su edad es más compatible con el evento volcánico del MaastrichtianoPaleoceno reportado en el interior del continente aproximadamente a la misma longitud geográfica (Ortega Gutiérrez 1980; Meza Figueroa et al., 2003; Cerca, 2004; Levresse et al., 2004) y que precede al pulso magmático del Eoceno tardío-Oligoceno temprano en la región central y occidental de la Sierra Madre del Sur.

Dentro de los cuerpos de ortogneis, agrupados en el Complejo Xolapa, se han reportado diferentes edades que indican que la mayor parte estos fueron emplazados y deformados entre el Jurásico Tardío y el Paleoceno (Guerrero-García, 1975; Robinson et al., 1989; MoránZenteno, 1992; Herrmann et al., 1994; Ducea et al., 2004a). Estas rocas se encuentran intrusionadas por los plutones no deformados lo que marca la edad mínima de su deformación y metamorfismo.

Dentro de los rasgos de deformación cenozoica posteriores a los eventos de deformación y metamorfismo del Complejo Xolapa, en el sector Zihuatanejo-Huatulco, destacan zonas de cizalla intensa que evolucionaron desde etapas de deformación cristal-plástica a deformación frágil (Ratchbacher et al., 1991; Riller et al., 1993, Tolson et al., 1993). Estas zonas de cizalla, sobre todo en su etapa cristal-plástica, preceden a la mayor parte de los intrusivos no deformados. Presentan indicadores de componentes de desplazamiento. normal y lateral izquierdo que han sido relacionados con la exhumación de la margen continental, y con un régimen generalizado de desplazamiento lateral cercanamente paralelo a la trinchera de Acapulco
(Ratschbacher et al., 1991; Riller et al., 1993; Tolson et al., 1993).

En la zona de cizalla ubicada al sur de Tierra Colorada se ha observado que un intrusivo granodiorítico de $34 \mathrm{Ma}$ de años corta a la zona milonítica, aunque la deformación continuó aún después del emplazamiento del plutón (Riller et al., 1993). En la región cercana al Golfo de Tehuantepec, Tolson (1998) reportó que la zona milonítica que limita en el norte al Complejo Xolapa, afecta al intrusivo de Huatulco de $29 \mathrm{Ma}$, lo que sugiere que en algunos sectores la deformación dúctil a lo largo de las zonas de cizalla continuó aún después del emplazamiento de los grandes plutones del Oligoceno.

Las causas que dieron origen a la exhumación de la margen continental desde Puerto Vallarta hasta el Golfo de Tehuantepec pueden ser diversas, pero seguramente procesos como el underplatting basáltico y el plutonismo silícico voluminoso, en combinación con el truncamiento continental y/o la erosión por subducción, jugaron un papel significativo. Morán-Zenteno et al. (1996) calcularon, con base en la geobarometría de aluminio total en hornblendas de los plutones paleógenos, que el levantamiento a lo largo de la línea de costa actual varió entre 13 y $20 \mathrm{~km}$. Tolson (1998) interpretó que esta exhumación en la región del Istmo de Tehuantepec pudo haber sido inducida por la migración a lo largo de la margen continental por un pliegue litosférico, producido por las diferencias de edad de la corteza subducida y por las características geométricas de la zona de subducción en esta región. Recientemente, Ducea et al., (2004b) obtuvieron datos de (UTh)/He que indican que la margen continental ha tenido una lenta exhumación desde el Mioceno temprano, lo que implica que el levantamiento principal se dio en el Oligoceno, después del emplazamiento de los plutones.

\section{Volcanismo y deformación durante el Eoceno- Mioceno medio en el interior del continente.}

En el Paleógeno se observa una migración del volcanismo del interior continental similar a la que experimentó el plutonismo de la costa, desde el Eoceno temprano, en la región de Michoacán, hasta el Mioceno medio, en el sureste de Oaxaca, aunque existen algunas excepciones a esta tendencia general (Figura $7 \mathrm{~b}$ y c).

En el oriente de Michoacán y el occidente de Guerrero, en la región ubicada entre Zihuatanejo y Ciudad Altamirano, existe una extensa cubierta de rocas volcánicas interestratificadas con depósitos sedimentarios continentales que cubre más de $20,000 \mathrm{~km}^{2}$ y que incluye la zona de la Presa del Infiernillo (Figura 2). Esta cubierta representa un importante pulso de volcanismo de composición intermedia y silícica para el que se han reportado algunas edades, aunque las relaciones estratigráficas generales no han sido estudiadas. Existen reportes de edades de $\mathrm{K}-\mathrm{Ar}$ desde $61.2 \pm 1.3$ (Pantoja-Alor, 1986) hasta $33 \mathrm{Ma}$ (Frank 
et al., 1992), aunque típicamente las edades reportadas de los derrames y los diques intermedios varían de 43 a 46 Ma (Figura 7b) (Kratzeisen et al., 1991; Frank et al., 1992: Garduño-Monroy et al., 1999). También existen algunos reportes de edades entre 38 y $48 \mathrm{Ma}$ en cuerpos aislados del norte de Guerrero y sur del Estado de México, los cuales representan un volumen menor que el de la región de la Presa del Infiernillo. Al norte de la zona volcánica de Zihuatanejo y Ciudad Altamirano, entre las longitudes 101 y $102^{\circ}$, se han reportado algunos cuerpos volcánicos y plutónicos con edades de $\mathrm{K}-$ Ar que varían entre 32 y $34 \mathrm{Ma}$ (Damon et al., 1981; Pasquaré et al., 1991). Esto representa una anomalía respecto a la tendencia decreciente de las edades hacia el este, ya que en la zona volcánica de Ciudad Altamirano-Zihuatanejo y en los plutones de la costa, las edades reportadas son en general más antiguas.

La actividad volcánica del Eoceno tardío y Oligoceno temprano que se desarrolló en las regiones de Morelos, Guerrero y occidente de Oaxaca, entre los meridianos $101^{\circ}$ y $97^{\circ}$ de long $\mathrm{W}$, representa uno de los mayores pulsos de actividad volcánica del interior continental. Esta actividad se concentró en una franja $\sim \mathrm{NW}-\mathrm{SE}$, de geometría paralela y edad contemporánea a la cadena de plutones de la margen continental (Figura 2).

En la región de Taxco-Huautla se distribuyen diferentes cuerpos volcánicos y plutónicos dominantemente silícicos (Figura 6) cuyas edades reportadas de $\mathrm{K}-\mathrm{Ar}$ y $\mathrm{Ar}-\mathrm{Ar}$ fluctúan principalmente entre los 38 y $31 \mathrm{Ma}$ (Figura 7c), aunque el mayor pulso de volcanismo parece haber ocurrido alrededor de los 35 Ma (De Cserna y Fries, 1981; Alaniz-Álvarez et al., 2002; Morán-Zenteno et al., 2004).

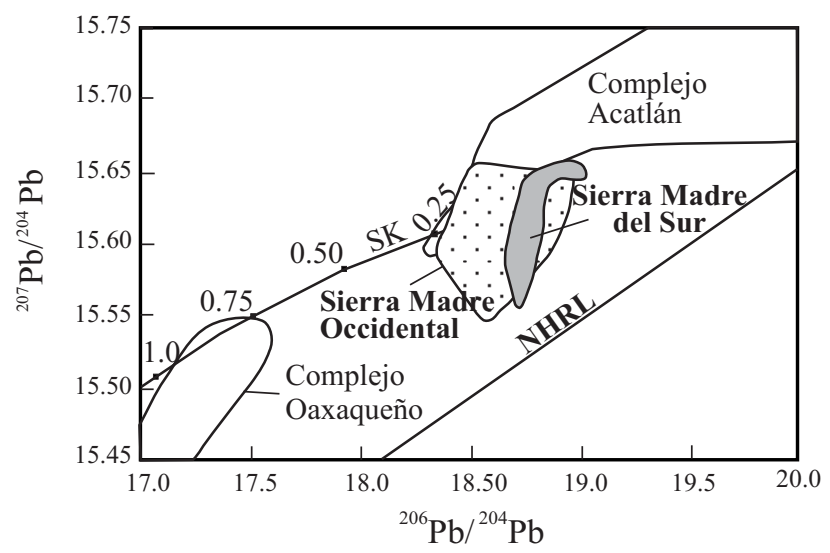

Figura 9. Distribución de los valores de las relaciones isotópicas de ${ }^{207} \mathrm{~Pb} /{ }^{204} \mathrm{~Pb}$ y ${ }^{206} \mathrm{~Pb} /{ }^{204} \mathrm{~Pb}$ para las rocas magmáticas del sur de México. Como referencia se muestran los campos de distribución típicos para las relaciones de $\mathrm{Pb}$ de los complejos Oaxaqueño y Acatlán, así como la distribución de los datos reportados para la Sierra Madre Occidental. Los datos fueron tomados de Martiny et al. (2000), Smith et al. (1996), Albrecht y Goldstein (2000). Las líneas de referencia indicadas son la curva de la evolución del plomo de dos etapas (Stacey and Kramers, 1975), graduada a intervalos de $250 \mathrm{Ma}$ (SK), y la línea de referencia del hemisferio norte (NHRL) (Hart, 1984)
Esta actividad volcánica es en general más reciente que la reconocida para el Paleógeno en Michoacán y el occidente de Guerrero y parcialmente contemporánea al plutonismo del sector de Acapulco-Tierra Colorada. Los centros volcánicos en esta región son del tipo de las calderas de colapso y campos de domos riolíticos. Esta actividad volcánica se encuentra principalmente relacionada a un evento tectónico de fallas de desplazamiento lateral izquierdo con orientación NW-SE que ha sido reconocido tanto en el área de Taxco (Alaniz et al., 2002), como en la zona de la caldera de Tilzapotla (Morán-Zenteno et al., 2004) (Figura 6). La actividad de estas fallas implica una dirección de máxima elongación NNW-SSE. En la región de Taxco, la extensión produjo la formación de una cuenca con elongación hacia el NW y la acumulación de una gruesa secuencia de depósitos fluviales del Eoceno (formación Chontalcuatlán) que subyace a la secuencia volcánica principal formada por ignimbritas y derrames riolíticos. Para el inicio del Oligoceno, se produjo un cambió tectónico en la región de Taxco con la activación de fallas laterales izquierdas de dirección al $\mathrm{N}$ y cambios en la cinemática de las fallas NWSE de izquierdas a derechas, lo que implica una dirección de la máxima elongación hacia el NE-SW.

Al oeste y noroeste de Taxco, en las sierras de la Goleta, Sultepec, Nanchititla y San Vicente, y al oriente de Valle de Bravo (De Cserna, 1982) (Figura 6), existen también centros volcánicos que parecen corresponder a calderas de colapso exhumadas para los cuales no se han reportado fechamientos que permitan limitar el periodo de la actividad volcánica. Por su composición predominantemente silícica y su estado de erosión pueden ser contemporáneas a los centros volcánicos de Taxco y Tilzapotla. También se han reportado zonas aisladas con unidades volcánicas silícicas al SE de la sierra de Huautla, en la región de Chiautla (Consejo de Recursos Minerales, 1998, Silva Romo et al., 2001) (Figura 6). El volumen expuesto de rocas silícicas en toda esta región es de alrededor de $3000 \mathrm{~km} 3$, lo que representa una estimación mínima con respecto al volumen original, ya que una gran parte de la cubierta volcánica parece haber sido removida por la erosión. Entre la región de Taxco y la región de las rocas plutónicas de Acapulco, las rocas volcánicas terciarias son más escasas. Para esta zona se han reportado preliminarmente algunos fechamientos de rocas volcánicas silícicas del final de Eoceno y principios del Oligoceno (Cerca et al., 2002; Campa Uranga et al., 2002).

En la región occidental de Oaxaca y sur de Puebla existe una extensa cubierta de rocas volcánicas dominantemente intermedias, que incluye también algunas unidades silícicas (Ferrusquía-Villafranca, 1976; Martiny et al., 2000). Esta secuencia de rocas volcánicas descansa en contacto transicional sobre depósitos fluviales y epiclásticos que revelan un episodio de sedimentación continental en cuencas intermontanas y fosas tectónicas. El estilo volcánico es de volcanes compuestos y centros monogenéticos que se encuentran en un avanzado estado de erosión. Para las 


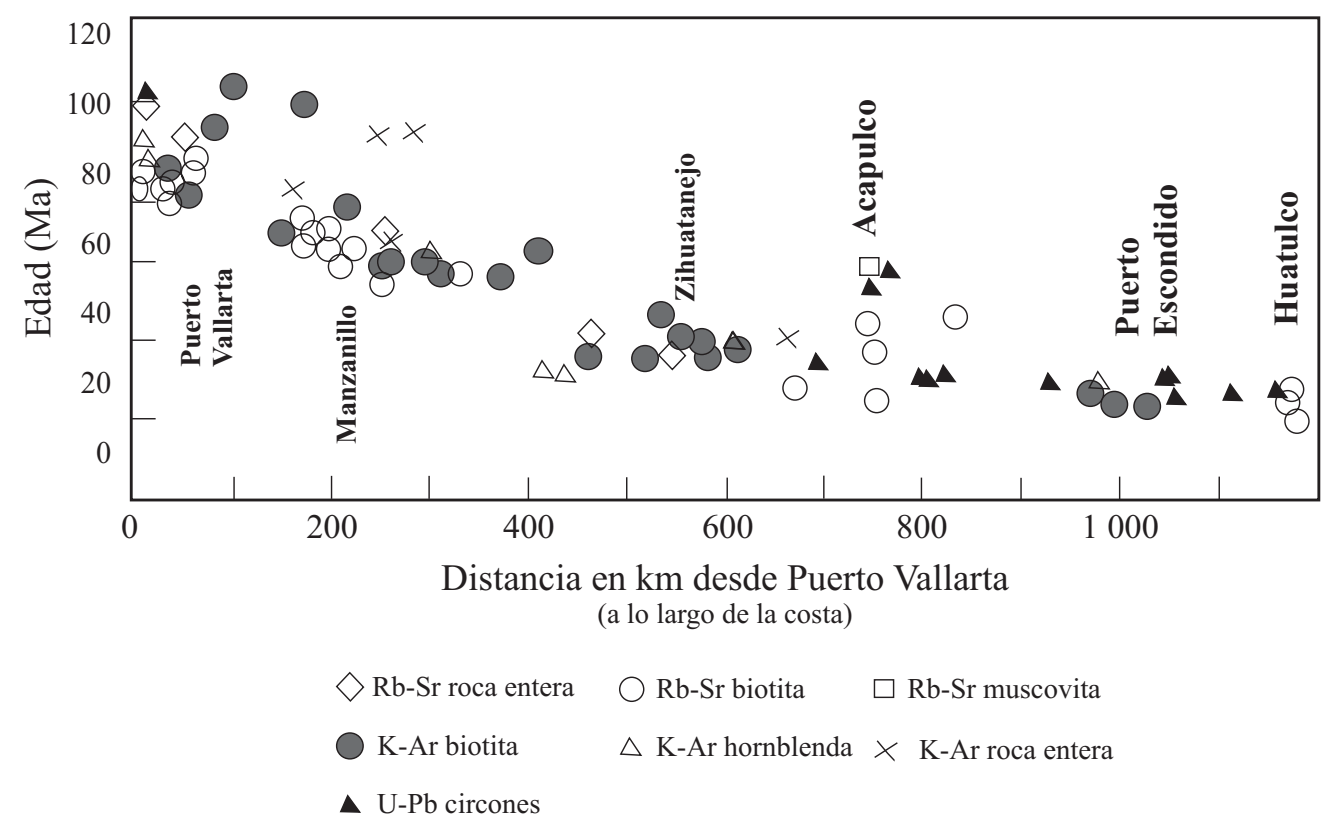

Figura 10. Gráfica que muestra la variación de las edades de los intrusivos a lo largo de la margen continental desde Puerto Vallarta, en el estado de Michoacán, hasta Huatulco, en el Estado de Oaxaca. Modificada de Schaaf et al. (1995) e incluyendo los datos de Ducea et al. (2004a).

rocas volcánicas de esta región se han reportado edades que varían principalmente entre 34-31 Ma (Martiny et al., 2000), aunque existen edades de hasta $26 \mathrm{Ma}$ (FerrusquíaVillafranca, 1992). Estas edades en general revelan una contemporaneidad parcial con las rocas más jóvenes del sector Taxco-Huautla. En el Valle de Oaxaca, el volcanismo alcanza edades de hasta $19 \mathrm{Ma}$ (Ferrsuquía-Villafranca, 1992; Urrutia-Fucugauchi y Ferrsuquía-Villafranca, 2001).

En la región occidental de Oaxaca el volcanismo también convivió con un episodio de desplazamiento lateral, primero asociado a fallas de orientación NW-SE y E-W, y después a fallas de laterales izquierdas de orientación al N (Martiny et al., 2002; Silva Romo et al., 2001). Estos episodios de fallamiento lateral no parecen ser exactamente contemporáneos a los de la región de Taxco-Huautla, lo que sugiere variaciones en tiempo y espacio de la deformación por fallas laterales.

Nieto-Samaniego et al. (2006) han interpretado una fase de deformación con extensión NE-SW, probablemente asociada al desplazamiento lateral izquierdo de las fallas $\mathrm{N}-\mathrm{S}$ en el occidente de Oaxaca. Esta fase de deformación tuvo efectos también en el Valle de Oaxaca en donde se desarrolló un semigraben de orientación NNW-SSE, para el cual se ha documentado sedimentación continental y volcanismo desde Oligoceno hasta el Mioceno temprano (Nieto Samaniego et al., 1995; Centeno García, 1988; Urrutia-Fucugauchi y Ferrusquía-Villafranca, 2001).

En el sureste de Oaxaca, en el sector Oaxaca-Salina Cruz, se han documentado secuencias volcánicas piroclásticas dominantemente silícicas que sobreyacen a secuencias sedimentarias continentales de conglomerados y areniscas (Ferrusquía-Villafranca, 1999; 2001). La cubierta volcánica y sedimentaria cenozoica en esta región cubre un área de aproximadamente $600 \mathrm{~km}^{2}$ (Figura 2). Las edades de $\mathrm{K}-\mathrm{Ar}$ reportadas por Ferrusquía-Villafranca (1992) fluctúan entre 13 y 17 Ma.

\section{Síntesis tectónica con relación a la cinemática de las placas en el entorno del sur de México}

La evolución tectónica del sur de México desde el Cretácico Tardío se caracteriza primero, por un evento orogénico de acortamiento con vergencia predominante hacia el oriente, el cual esta relacionado en tiempo y estilo de deformación con la orogenia Laramide. Posteriormente, a partir del Eoceno, se desarrolló de un régimen tectónico caracterizado por fallas laterales cuyas direcciones preferenciales tanto de las trazas principales como del acortamiento y extensión asociados variaron en el tiempo, pero también en espacio.

El origen de los esfuerzos que produjeron el acortamiento regional laramídico es todavía objeto de controversia. En la interpretación tradicional, el acortamiento comienza al inicio del Cretácico Tardío en el terreno Guerrero y se propaga hacia el oriente en el Cretácico Tardío y aún hasta el Eoceno en la región de Veracruz (i. e. Salinas-Prieto et al., 2000; Eguiluz de Antuñano et al., 2000; NietoSamaniego et al., 2006). La hipótesis más aceptada sobre las causas de este evento de deformación a lo largo de la Cordillera Norteamericana, es el acoplamiento mecánico y la tracción que resultaron de la subducción de la placa de Farallón en una posición subhorizontal bajo la corteza de la placa de Norteamérica. Esta posición ha sido atribuida al 
aumento de la velocidad de convergencia entre las placas de Norteamérica y de Farallón (Engebretson, et al., 1985; Dickinson et al., 1988; Bird, 1988; Bunge y Grand, 2000; Saaleby, 2003). El inicio del acortamiento documentado en el suroeste de México coincide con un periodo de aumento de la velocidad absoluta de Norteamérica hacia el sur entre 88 y 80 Ma (Beck y Housen, 2003).

Existen problemas para la aplicación de este modelo desarrollado en la Cordillera Norteamericana en el sur de México. La existencia de actividad magmática de arco durante y posteriormente a la deformación en una zona cercana a la paleotrinchera inferida (zona del bloque Jalisco) no parecen registrar un reducción substancial del ángulo de subducción ya que esto habría inhibido el magmatismo de arco como ha sido mostrado en modelos térmicos recientes (English et al., 2003).

Otra inconsistencia que comienza a delinearse a partir de los trabajos recientes es la presencia de magmatismo postorogénico (González-Partida et al., 2003) al poniente del núcleo metamórfico antiguo (complejos Oaxaqueño y Acatlán), contemporáneo con el acortamiento observado en el oriente de la Sierra Madre del Sur (Eguiluz de Antuñano et al., 2000).

Una posibilidad alternativa, sugerida por otros autores para explicar la deformación por acortamiento, implica un escenario tectónico más complejo con la acreción de un arco insular asociado a una zona de subducción con vergencia al oeste y la clausura de una cuenca oceánica (Campa y Coney, 1983; Monod et al., 1994; Salinas-Prieto et al., 2000; Freydier et al., 1996). Este modelo ha sido cuestionado por Elías-Herrera y Ortega-Gutiérrez (1998), señalando que no hay evidencias de asociaciones petrotectónicas típicas de suturas, además de la clara influencia continental en sedimentos y magmas del terreno Guerrero en la vecindad de su contacto tectónico con los terrenos más antiguos del sur de México.

Además de estas consideraciones, el estilo estructural observado en el sur de México involucra deformación de cobertura cuyos patrones cruzan las fronteras de los supuestos bloques (terrenos de basamento), al menos al occidente del Complejo Acatlán (ver Lang et al., 1996; Salinas-Prieto et al., 2000; Cabral-Cano et al., 2000 a y b; Elías-Herrera et al., 2000). Estas observaciones contrastan con la idea de que la geometría de la deformación debería ser fuertemente dependiente de la geometría de los bloques alóctonos involucrados, puesto que durante la acreción de estos terrenos, la deformación se concentra en los límite, aunque una parte de ella puede transmitirse y distribuirse hacia dentro de los terrenos involucrados dependiendo de la intensidad, del tiempo transcurrido y de sus propiedades reológicas. Las fallas mayores preexistentes cortan la corteza y crean zonas de cizalla que pueden servir como limites de estilos estructurales.

El episodio magmático del Maastrichtiano-Paleoceno sucede a la deformación laramídica en el occidente y centro de la Sierra Madre del Sur (González-Partida et al., 2003).
Las características geoquímicas de estas rocas indican que su origen se relaciona a un magmatismo de arco asociado a un límite convergente, cuya orientación no se conoce con precisión. La dirección de los pliegues y cabalgaduras laramídicos sugiere una margen continental del Pacífico con orientación aproximada N-S. Sin embargo, la distribución de las rocas del magmatismo de arco MaastrichtianoPaleoceno favorece una orientación NW-SE (Figura 7a).

No se sabe con certeza cuándo terminó la deformación Laramide y cuándo comenzó el régimen lateral generalizado que predominó durante el Cenozoico, pero en el Eoceno tardío se registra una actividad tectónica expresada principalmente en fallas laterales izquierdas de orientación NW-SE y E-W. Ésta se reconoce principalmente en la parte norte-central de la Sierra Madre del Sur y a lo largo de la margen continental actual, entre Zihuatanejo y Puerto Escondido (Figuras 6 y 10) (Ratschbacher et al., 1991; Morán-Zenteno, et al., 1999; Alaniz-Álvarez, 2002). Hacia el inicio del Oligoceno el fallamiento lateral izquierdo, expresado por la presencia de fallas E-W, prevalecía en la región de Huatulco (Tolson et al., 1993). En la parte-norte central de la Sierra Madre del Sur, sin embargo, se puede documentar para inicios del Oligoceno un cambio en la cinemática de la deformación que provocó la reactivación de fallas con orientación N-S y desplazamiento también lateral izquierdo en fallas de orientación similar, en sectores ubicados desde el norte de Guerrero hasta la parte central de Oaxaca (Alaniz-Álvarez et al., 2002; Silva Romo et al., 2001). Considerando las heterogeneidades del basamento premesozoico y la existencia de algunas zonas de debilidad preexistentes en esta región, es difícil establecer las condiciones de esfuerzo en las que ocurrieron estos cambios, pero las direcciones de extensión asociadas a cada régimen variaron de NNW-SSE a NE-SW. De acuerdo a Nieto Samaniego, et al. (2006), a esta última dirección de extensión se asocia la reactivación de la falla de Oaxaca, lo que produjo la formación de un semigraben de orientación NNW-SSE (Figura 11). Meschede et al. (1996), basados en la medición de planos falla y estrías a escala de afloramiento, definieron diferentes grupos de fallas que parecen responder a paleotensores de esfuerzo activos en diferentes tiempos en el sur de México. Estos autores infirieron que para el periodo entre 70 a 40 Ma la orientación de $\sigma_{1}$ era aproximadamente $\mathrm{E}-\mathrm{W}$, con una orientación subhorizontal de $\sigma_{3}$, las cuales definieron una deformación dominante de fallas de desplazamiento lateral que incluirían las zonas de cizalla de orientación NW-SE cercanas a la costa. Sin embargo, la cronología y direcciones sugeridas por estos autores no son totalmente compatibles con la cronología y la cinemática inferida del estudio de las grandes estructuras en el interior del continental, como las documentadas desde el sector de Taxco a Huajuapan en la parte norte de la Sierra Madre del Sur, las cuales tuvieron actividad significativa después de los $40 \mathrm{Ma}$ (Alaniz-Álvarez et al., 2002; Martiny et al., 2002, Morán-Zenteno et al. 2004).

El truncamiento continental, la migración del mag- 


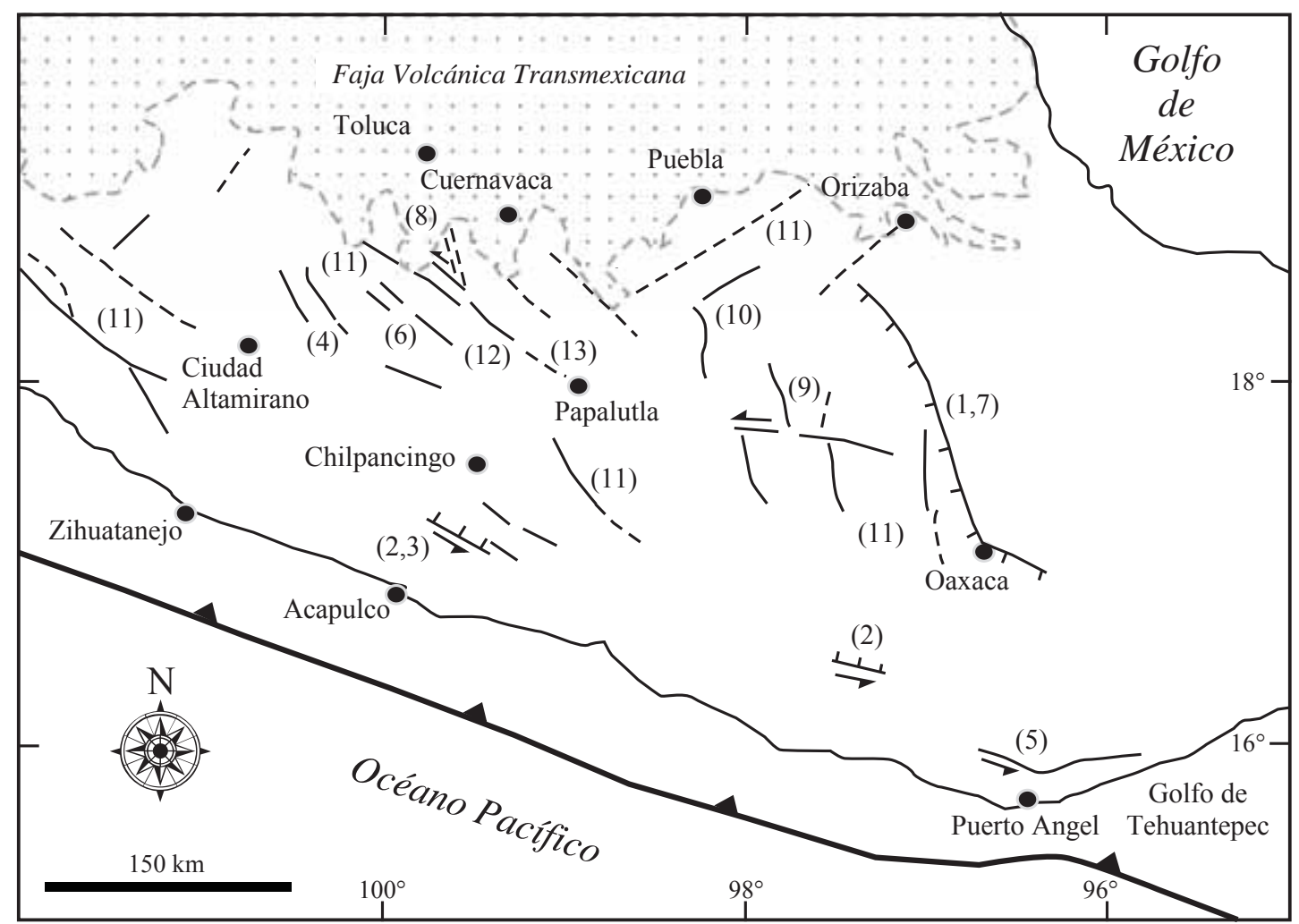

Figura 11. Distribución de los principales rasgos tectónicos del Cenozoico reconocidos en el sur de México. Se incluyen rasgos que han sido descritos en sus detalles geométricos y cinemáticos, pero también se incluyen lineamientos evidentes señalados en los mapas de escala 1:250,000 del Consejo de Recurso Minerales (hojas Ciudad Altamirano E 14-4, Cuernavaca E 14-5, Chilpancingo E 14-8, Oaxaca E14-9, Orizaba E 14-6), cuyos detalles cinemáticos y geométricos no se conocen. En la mayor parte de los lineamientos no se incluyen indicaciones de desplazamiento relativo debido a que se han documentado historias cinemáticas complejas. En los que se muestra, el desplazamiento relativo se refiere al desplazamiento principal documentado para el Eoceno tardío u Oligoceno temprano. Los números indican las fuentes de la que fue tomada la información: 1) Centeno-García (1998) 2) Ratschbacher et al. (1991); 3) Riller et al. (1993); 4) Jansama y Lang (1997); 5) Tolson (1998); 6) Cabral-Cano et al. (2000); 7) Alaniz-Álvarez et al. (1994); 8) Alaniz-Álvarez et al. (2002); 9) Martiny et al. (2002); 10) Silva-Romo et al. (2002); Rivera-Carranza et al. (1997); 11) Morán-Zenteno et al. (2004); 12) Cerca (2004).

matismo hacia el SE y el desarrollo de zonas de falla con componente lateral izquierdo, han sido vinculadas en los últimos años al desplazamiento del bloque Chortis a lo largo de la margen continental, desde una posición inicial entre Puerto Vallarta y el Istmo de Tehuantepec (Figura 3a) (Schaaf et al., 1995; Meschede et al., 1996; Morán-Zenteno et al., 1999). La extinción del magmatismo en la Sierra Madre del Sur en el Eoceno-Oligoceno y su reaparición en la posición de la FVTM a partir del Mioceno, se relacionan a una cambio en la geometría de la placa subducida. La idea dominante hasta ahora ha sido que el cambio en la geometría es el resultado del paso del punto triple trinchera-trinchera-transforme que acompañó al bloque Chortis, y también que se relaciona con la exhumación de la margen continental (Schaaf et al., 1995; Morán-Zenteno et al., 1996; Tolson, 1998). La posición del bloque de Chortis frente a las costas del sur de México fue sugerida previamente por otros autores como una necesidad geométrica en las reconstrucciones paleogeográficas relacionadas con la evolución de la placa del Caribe (Malfait y Dinkelman, 1972; Anderson y Schmidt, 1983; Ross y Scotese, 1988).
Aunque esta interpretación es compatible con las tendencias geocronológicas y la cinemática observada en las fallas de la región, presenta algunos problemas si se analiza desde la perspectiva de la cinemática de las placas en la región y los efectos esperados a lo largo de la margen continental. Quizá los problemas principales tienen que ver con la aparente incompatibilidad entre el desplazamiento del bloque Chortis y la rotación de la placa del Caribe desde el Eoceno hasta el reciente y la no concordancia de los rasgos tectónicos o petrotectónicos antiguos entre Honduras y México. El límite norte de la placa del Caribe está conformado por el lineamiento y fosa del Cayman y así como por el sistema de fallas Polochic-Motagua. La forma de este límite norte, así como la edad de los sedimentos y el patrón de las anomalías magnéticas de la Fosa del Caimán, han permitido estimar un desplazamiento de aproximadamente $1100 \mathrm{~km}$ de la placa del Caribe hacia el este con respecto a Norteamérica desde el Eoceno medio (Rosencrantz y Sclater, 1986). La cronología de este desplazamiento está también definida por el cambio de la geometría del límite norte de la placa del Caribe después de la colisión de Cuba 
contra la plataforma de las Bahamas. La trayectoria promedio de la placa del Caribe con respecto a Norteamérica desde el Eoceno estaría definida, de acuerdo a la tendencia de la fosa del Cayman, según un polo de rotación promedio ubicado en el hemisferio sur (Pindell et al., 1988). Solo en el segmento occidental del límite norte de la placa del Caribe, correspondiente a las fallas Polochic y Motagua, se observa una ligera concavidad hacia el norte que rompe con la tendencia general de la fosa del Cayman.

El desplazamiento del bloque de Chortis en varias interpretaciones es hacia el sureste (Ross y Scotese, 1988, Pindell et al., 1988, Schaaf et al., 1995) y no hacia el este como parece ser para el resto de la placa del Caribe. Si el bloque de Chortis hubiera estado presente frente a la costa actual de Guerrero y Oaxaca en el Eoceno, y además hubiera sido parte de la placa del Caribe, los efectos esperados de su desplazamiento serían los de deformación por transpresión a lo largo de la margen continental. Evidencias de transpresion compatible con un régimen tectónico de desplazamiento lateral izquierdo han sido documentadas en el interior continental en un episodio previo al Eoceno (Cerca et al., 2004) y para la región de Chiapas desde el Mioceno (Meneses-Rocha, 1991; Guzmán-Speciale y Meneses-Rocha, 2000). Sin embargo, las observaciones estructurales de las zonas de cizalla semiparalelas a la costa en Guerreo y Oaxaca occidental son más compatibles con un mecanismo de transtensión (Ratschbacher, et al., 1991, Tolson et al., 1993; Riller et al., 1993; Herrmann et al., 1994; Tolson, 1998). Otra limitante de este modelo es la existencia de una secuencia solo ligeramente deformada de sedimentos marinos en el Golfo de Tehuantepec, cuya edad varía desde el Cretácico Tardío hasta el Holoceno (Sánchez Barreda, 1981), lo que no parece compatible con el movimiento del bloque de Chortis en condiciones de transpresión. Un problema que ha sido señalado desde hace algunos años es el de el desplazamiento significativamente menor (130-170 km) que se ha documentado a lo largo del sistema de fallas Polochic-Motagua (e. g. Burkart et al., 1987; Donnelly et al., 1990; Guinta et al., 2002 y referencias incluidas) comparado con el desplazamiento de $\sim 1100 \mathrm{~km}$ estimado en la fosa del Caimán (Rosencrantz y Sclater, 1986).

Keppie y Morán-Zenteno (2005) han evaluado la posibilidad de que el bloque de Chortis proceda de una posición al oeste-suroeste de su posición actual, de acuerdo a una rotación horaria, tal como lo sugiere la configuración de la fosa del Cayman (límite norte de la placa del Caribe) (Figura $3 \mathrm{a}$ y b). Esta paleoposición del bloque Chortis puede explicar el desplazamiento restringido documentado en el sistema de fallas Polochic-Motagua, el cual habría ocurrido después de un primer periodo de desplazamiento relativo a lo largo de la Fosa del Cayman, antes de que entrara en contacto el bloque Chortis con el segmento chiapaneco del bloque Maya. El desarrollo del sistema de fallas Polochic-Motagua con concavidad al norte sería resultado de la interacción tectónica transpresiva más re- ciente (a partir del Mioceno) entre el bloque Chortis y el segmento chiapaneco del bloque Maya (Guzmán-Speciale y Meneses-Rocha, 2000). La trayectoria alternativa del bloque Chortis tendría como consecuencia que la cresta de Tehuantepec entrara en contacto con la costa del sur de México después del Eoceno y que eventualmente produjera un cambio en la geometría de la subducción como la que se observa actualmente. Las limitantes que presenta este modelo, surgen de la necesidad de explicar el truncamiento de la margen occidental del sur de México y la tectónica de fallas laterales observadas en Guerrero y Oaxaca con edades del Eoceno y Oligoceno. En este caso se tendrían que introducir elementos que explicaran una intensa erosión por subducción y probablemente una convergencia oblicua que facilitara el desarrollo de componentes de desplazamiento lateral izquierdo. Keppie y Morán-Zenteno (2005) han sugerido que la erosión por subducción al noroeste del Istmo de Tehuantepec y la exhumación de la margen continental después de los principales eventos de plutonismo, pudieron haber sido producidos por el arribo de la cadena de montes marinos cuya contraparte se encuentra hoy entre las fracturas Molokai y Clarión. Aunque este modelo, en combinación con la colisión la cresta de Tehuantepec, explica el cambio en la geometría de la placa subducida y la erosión por subducción, deja dudas sobre el patrón de migración del magmatismo hacia el sureste. De acuerdo con los vectores de movimiento relativo calculados por Schaaf et al. (1995), con base en los polos de rotación de Engebretson et al. (1985) y de Pindell et al. (1988), la convergencia oblicua de la placa de Farallón pudo haber favorecido el desarrollo de fallas laterales izquierdas de orientación NW. Otro factor que seguramente jugó un papel importante en el desarrollo del fallamiento lateral en el sur de México es el comportamiento heterogéneo de la geometría de la subducción al norte y al sur de la FVTM actual. Mientras en la región de la Mesa Central, la placa subducida iniciaba un retroceso (Nieto Samaniego et al., 1999), en el sur la placa disminuía su ángulo de subducción (Morán-Zenteno et al., 1999). Este último factor pudo también haber tenido influencia en el patrón de migración magmático observado.

Finalmente, aunque los datos acumulados hasta ahora no permiten descartar completamente los modelos tradicionales del acortamiento Laramídico en el Cretácico Tardío y Cenozoico temprano, o de la posición del bloque Chortis frente a la margen continental del sur de México y su desplazamiento hacia el sureste durante el Cenozoico, muchos de los rasgos observados e interpretaciones planteadas en este trabajo pueden admitir modelos alternativos que requieren de una valoración más detallada y profunda.

\section{Agradecimientos}

M. Cerca agradece a CONACYT una beca de estudios y a Luca Ferrari por estimulantes discusiones sobre el sur 
de México. La revisión crítica de dos árbitros anónimos ayudó a mejorar en gran medida este trabajo. Los estudios realizados por D. Morán-Zenteno en la parte norte-central de la Sierra Madre del Sur fueron financiados por el programa de apoyo a proyectos de investigación e innovación tecnológica (PAPIIT) de la UNAM (proyectos IN 104802-2 e IN 120502-2).

\section{Referencias bibliográficas}

Aguilera-Franco, N., Hernández-Romano, U., 2004, CenomanianTuronian facies succession in the Guerrero-Morelos basin, southern México: Sedimentary Geology, 170, 135-162.

Alaniz-Álvarez, S. A., Nieto-Samaniego, Á. F., Ortega-Gutiérrez, F., 1994, Structural evolution of the Sierra de Juárez mylonitic complex, state of Oaxaca, México: Revista Mexicana de Ciencias Geológicas, $11,147-156$

Alaniz-Álvarez, S. A., Nieto-Samaniego, Á. F., Morán-Zenteno, D. J., Alba-Aldave, L., 2002, Rhyolitic volcanism in extension zone associated with strike-slip tectonics in the Taxco region, southern Mexico: Journal of Volcanology and Geothermal Research, 118, $1-14$.

Albrecht, A., Goldstein, S. L., 2000, Effects of basement composition and age on silicic magmas across an accreted terrane-Precambrian crust boundary, Sierra Madre Occidental, Mexico: Journal of South American Earth Sciences, 13, 255-273.

Altamira-Areyán, A., 2002, Las litofacies y sus implicaciones de la cuenca sedimentaria Cutzamala-Tiquicheo, estados de Guerrero y Michoacán, México: México, Universidad Nacional Autónoma de México, Instituto de Geología, Posgrado en Ciencias de la Tierra, Tesis de maestría, $79 \mathrm{p}$

Anderson, T. H., Schmidt, V. A., 1983, The evolution of middle America and the Gulf of Mexico-Caribbean sea region during Mesozoic time: Geological Society of America Bulletin, 94, 941-966.

Bandy, W., Mortera-Gutiérrez, C., Urrutia-Fucugauchi, J., Hilde, T. W. C., 1995, The subducted Rivera-Cocos plate boundary: where is it, what is it, and is its relationship to the Colima rift?: Geophysical Research Letters, 22, 3075-3078.

Barrier, E., Velasquillo, L., Chávez, M., Goulon, R., 1998, Neotectonic evolution of the Isthmus of Tehuantepec (southeastern Mexico) Tectonophysics, 287, 77-96.

Beck, M. E., Housen, B. A. 2003. Absolute velocity of North America during the Mesozoic from paleomagnetic data: Tectonophysics, $377,33-54$

Bellon, H., Maury, R. C., Stephan, J. F., 1982, Dioritic basement, site 493: petrology, geochemistry, and geodynamics: Initial Reports of the Deep Sea Drilling Project, LXVI, 723-731.

Benammi, M., Centeno-García, E., Martínez-Hernández, E., MoralesGámez, M., Tolson, G., Urrutia-Fucugauchi, J., 2005, Presencia de dinosaurios en la Barranca Los Bonetes en el sur de México (Región de Tiquicheo, Estado de Michoacán) y sus implicaciones cronoestratigráficas: Revista Mexicana de Ciencias Geológicas, 22(3), 429-435.

Bird, P., 1988, Formation of the Rocky Mountains western United States: a continuum computer model: Science, 239, 1501-1507.

Bird, P., 1998, Kinematic history of the Laramide orogeny in latitudes $35^{\circ}$ - $49^{\circ} \mathrm{N}$, western United States: Tectonics, 17, 780-801.

Bunge, H. P., Grand, S. T., 2000, Mesozoic plate-motion history below the northeast Pacific Ocean from seismic images of the subducted Farallon slab: Nature, 405, 337-340.

Burkart, B., Deaton, B. C., Dengo, C., Moreno, G., 1987, Tectonic wedges and offset Laramide structures along the Polochic fault of Guatemala and Chiapas, Mexico: reaffirmation of large Neogene displacement: Tectonics, 6, 411-422.

Cabral-Cano, E., Lang, H. R., Harrison, C. G. A., 2000a, Stratigraphic assessment of the Arcelia-Teloloapan area, southern Mexico: impli- cations for southern Mexico's post-Neocomian tectonic evolution: Journal of South American Earth Sciences, 13, 443 - 457.

Cabral-Cano, E., Draper, G., Lang, H. R., Harrison, C. G. A., 2000b, Constraining the late Mesozoic and early Tertiary tectonic evolution of southern Mexico: structure and deformation history of the Tierra Caliente region, southern Mexico: Journal of Geology, 108, 427-446.

Campa, M. F., Coney, P. J., 1983, Tectono-stratigraphic terranes and mineral resource distributions in Mexico: Canadian Journal of Earth Sciences, 20, 1040-1051.

Campa, M. F., Ramírez, J., 1979, La evolución geológica y la metalogénesis del noroccidente de Guerrero. Plan Piloto Proyecto de Recursos Naturales de Guerrero: Taxco, Universidad Autónoma de Guerrero, Serie Científica Técnica, 1, 100 p.

Campa, M. F., Oviedo, A., Tardy, M., 1976, La cabalgadura laramídica del dominio volcanico-sedimentario (Arco de Alisitos-Teloloapán) sobre el miogeosinclinal mexicano en los límites de los estados de Guerrero y México, en III Congreso Latinoamericano de Geología, Resúmenes: México, D. F., Universidad Nacional Autónoma de México, Instituto de Geología, 23.

Campa-Uranga, M. F., García-Díaz, J. L., Bustamante-García, J., Torreblanca-Castro, T. de J., Aguliera-Martínez, M. A., Vergara Martínez, A., 1997, Carta geológico-minera de la hoja Chilpancingo. Escala 1:250.000 (E14-8): Pachuca, Hidalgo, Consejo de Recursos Minerales, 1 mapa.

Campa-Uranga, M. F., Fitz-Díaz, E., Martínez-Hernández, E., 2002, Revisión de la edad de la Formación Oapan y su significado en el graben de San Agustín Oapan y el sinclinorio de Zacango, estado de Guerrero, en XII Congreso Nacional de Geoquímica, Resúmenes: Actas INAGEQ, 8, 165.

Centeno-García, E., 1988, Evolución estructural de la falla de Oaxaca durante el Cenozoico: México, Universidad Nacional Autónoma de México, Facultad de Ciencias, Tesis de maestría, 156 p.

Centeno-García, E., García, J. L., Guerrero-Suástegui, M., RamírezEspinosa, J., Salinas-Prieto, J. C., Talavera-Mendoza, O., 1993a, Geology of the southern part of the Guerrero terrane, Ciudad Altamirano-Teloloapan area, en Ortega-Gutiérrez, F., CentenoGarcía, E., Morán-Zenteno, D. J., Gómez-Caballero, A. (eds.), First Circum-Pacific and Circum-Atlantic Terrane Conference, Terrane geology of southern Mexico. Guidebook of Field trip B: Guanajuato, Gto., Universidad Nacional Autónoma de México, Instituto de Geología, 22-33.

Centeno-García, E., Ruiz, J., Patchett, P. J., Ortega-Gutiérrez, F., 1993b, Guerrero terrane-Its role in the evolution southern Cordillera from new geochemical data: Geology, 21, 419-423.

Cerca, M., 2004, Deformación y magmatismo Cretácico tardío - Terciario temprano en la zona de la Plataforma Guerrero Morelos: Juriquilla, Qro., Universidad Nacional Autónoma de México, Centro de Geociencias, Posgrado en Ciencias de la Tierra, 175 p., 1 mapa.

Cerca, M., Ferrari, L., López-Martínez, M., Iriondo A., 2002, Cartografía geológico-estructural de la zona oriental de la Plataforma Guerrero-Morelos, en III Reunión Nacional de Ciencias de la Tierra, Resúmenes: GEOS, 22(2), 179-180.

Cerca, M., Ferrari, L., Bonini, M., Corti, G., Manetti, P., 2004, The role of crustal heterogeneity in controlling vertical coupling during Laramide shortening and the development of the Caribbean - North American transform boundary in southern Mexico: insights from analogue models, en Grocott, J., Taylor, G., Tikoff, B. (eds).,Vertical coupling and decoupling in the lithosphere: London, Geological Society, Special Publication, 227, 117-140.

Chapell, B. W., White, A. J. R., 1974, Two contrasting granite types: Pacific Geology, 8, 173-174.

Damon, P. E., Shafiqullah, M., Clark, K. F., 1981, Geochronology of the porphyry copper deposits and related mineralization of Mexico: Canadian Journal of Earth Sciences, 20, 1052-1071.

De Cserna, Z., 1982, Hoja Tejupilco 14Q-g(9), con resumen de la hoja Tejupilco, estados de Guerrero, México y Michoacán: México, D. F., Universidad Nacional Autónoma de México, Instituto de Geología, Carta Geológica de México, Serie de 1:100,000, 1 mapa con texto explicativo, $28 \mathrm{p}$. 
De Cserna, Z., Ortega-Gutiérrez, F., Palacios-Nieto, M., 1980, Reconocimiento geológico de la parte central de la cuenca del alto Río Balsas, estados de Guerrero y Puebla, en V Convención Geológica Nacional, Libro Guía de la excursión geológica a la parte central de la cuenca del alto Río Balsas, estados de Guerrero y Puebla: México, Sociedad Geológica Mexicana, 2-33.

De Cserna, Z., Fries, C. Jr., 1981, Hoja Taxco 14Q-h(7), con resumen de la hoja Taxco, estados de Guerrero, México y Morelos: México, D. F., Universidad Nacional Autónoma de México, Instituto de Geología, Carta Geológica de México, Serie de 1:100,000, 1 mapa con texto explicativo, $47 \mathrm{p}$.

Delgado-Argote, L., Carballido-Sánchez, E., 1990, Análisis tectónico del sistema transpresivo neogénico entre Macuspana, Tabasco, y Puerto Ángel, Oaxaca: Revista del Instituto de Geología, Universidad Nacional Autónoma de México, 9, 21-32.

Delgado-Argote, L., López-Martínez, M., Cork, D., Hall, M., 1992, Geologic framework and geochronology of ultramafic complexes of southern Mexico: Canadian Journal of Earth Sciences, 29, 1590 -1604 .

DeMets, C., Stein, S., 1990, Present-day kinematics of the Rivera plate and implications for tectonics in southwestern Mexico: Journal of Geophysical Research, 95, 21931-21948.

DeMets, C., Wilson, D. S., 1997, Relative motions of the Pacific, Rivera, North American, and Cocos plates since $0.78 \mathrm{Ma}$ : Journal of Geophysical Research, 102, 2789-2806. DeMets, C., Traylen, S., 2000, Motion of the Rivera plate since 10 Ma relative to the Pacific and North American plates and the mantle: Tectonophysics, 318, 119-159.

Dickinson, W. R., Lawton, T. F., 2001, Carboniferous to Cretaceous assembly and fragmentation of Mexico: Geological Society of America Bulletin, 113, 1142-1160.

Dickinson, W. R., Klute, M. A., Hayes, M. J., Janecke, S. U., Lundin, E. R., McKittrick, M. A., Olivares, M. D., 1988, Paleogeographic and paleotectonic setting of Laramide sedimentary basins in the central Rocky Mountain region: Geological Society of America Bulletin, 100, 1023-1039.

Donnelly, T. W., Horne, G. S., Finch, R. C., López-Ramos, E., 1990, Northern central America; the Maya and Chortis blocks, en Dengo, G., Case, J. E. (eds.), The Caribbean region: Boulder, Colorado, Geological Society of America, The Geology of North America, H, 37-76.

Ducea, M. N., Gehrels, G. E., Shoemaker, S., Ruiz, J., Valencia, V. A., 2004a, Geologic evolution of the Xolapa Complex, southern Mexico. Evidence from U-Pb Zircon geochronology: Geological Society of America Bulletin, 116, 1016-1025

Ducea, M., Valencia, V. A., Shoemaker, S., Reiners, P. W., DeCelles, P. G., Campa, M. F., Morán-Zenteno, D., Ruiz, J., 2004b, Rates of sediment recycling beneath the Acapulco trench: constraints from (U-Th)/He thermochronology: Journal of Geophysical Research, 109(B9), B09404 doi 10.1029/2004JB003112, 1-11.

Eguiluz de Antuñano, S., Aranda-García, M., Marrett, R., 2000, Tectónica de la Sierra Madre Oriental, México: Boletín de la Sociedad Geológica Mexicana, 53(1), 1-26.

Elías-Herrera, M., Ortega-Gutiérrez, F., 1998, The early Cretaceous Arperos oceanic basin (western Mexico). Geochemical evidence for an aseismic ridge formed near a spreading center - Comment: Tectonophysics, 292, 321-326

Elías-Herrera, M., Sánchez-Zavala, J. L., Macías-Romo, C., 2000, Geologic and geochronologic data from the Guerrero terrane in the Tejupilco area, southern Mexico: new constraints on its tectonic interpretation: Journal of South American Earth Sciences, $13,355-375$

Engebretson, D. C., Cox, A., Gordon, R. G., 1985, Relative plate motions between oceanic and continental plates in the Pacific basin: Boulder, Colorado, Geological Society of America, Special Paper, 206, 64 p.

English, J. M., Johnston, S. T., Wang, K., 2003, Thermal modeling of the Laramide orogeny: testing the flat-slab subduction hypothesis: Earth and Planetary Science Letters, 214, 619-632.

Ferrari, L., Pasquarè, G., Venegas, S., Castillo, D., Romero, F., 1994,
Regional tectonics of western Mexico and its implications for the northern boundary of the Jalisco block: Geofísica Internacional, $33,139-151$.

Ferrari, L., López-Martínez, M., Aguirre-Díaz, G., Carrasco-Núñez, G., 1999, Space-time patterns of Cenozoic arc volcanism in central Mexico: from the Sierra Madre Occidental to the Mexican Volcanic Belt: Geology, 27, 303-306. Ferrari, L., López-Martínez, M., Rosas-Elguera, J., 2002, Ignimbrite flare up and deformation in the southern Sierra Madre Occidental, western Mexico: implications for the late subduction history of the Farallon plate: Tectonics, $21(4), 17 / 1-17 / 24$.

Ferrusquía-Villafranca, I., 1976, Estudios geológico-paleontológicos en la región Mixteca, Parte 1: Geología del área TamazulapanTeposcolula-Yanhuitlán, Mixteca Alta, estado de Oaxaca, México: Boletín del Instituto de Geología, Universidad Nacional Autónoma de México, 97, 185 p.

Ferrusquía-Villafranca, I., 1992, Contribución al conocimiento del Cenozoico en el sureste de México y de su relevancia en el entendimiento de la evolución regional, en III Congreso Geológico de España y VIII Congreso Latinoamericano de Geología, Actas de las sesiones científicas: Salamanca, 4, 40-44.

Ferrusquía-Villafranca, I., 1999, Contribución al conocimiento de Oaxaca, México-El área Laollaga-Lachivizá: Boletín del Instituto de Geología, Universidad Nacional Autónoma de México, 110, 103 p., 2 láms.

Ferrusquía-Villafranca, I., 2001, Contribución al conocimiento geológico de Oaxaca. México-El área de Nejapa de Madero: Boletín del Instituto de Geología, Universidad Nacional Autónoma de México, 111,100 p., 3 láms.

Frank, M., Kratzeisen, M., Negendank, J. F. W., Boehnel, H., 1992, Geología y tectónica en el terreno Guerrero (México-Sur), en III Congreso Geológico de España y VIII Congreso Latinoamericano de Geología, Actas de las sesiones científicas: Salamanca, España, 4, 290-293.

Freydier, C., Martínez-R., J., Lapierre, H., Tardy, M., Coulon, C., 1996, The early Cretaceous Arperos oceanic basin (western Mexico). Geochemical evidence for an aseismic ridge formed near a spreading center: Tectonophysics, 259, 343-367.

Fries, C., Jr., 1960, Geología del estado de Morelos y de partes adyacentes de México y Guerrero, región central meridional de México: Boletín del Instituto de Geología, Universidad Nacional Autónoma de México, 60, 236 p., 5 láms.

Fries, C., Jr., 1966, Hola Cuernavaca 14Q-h(8), con resumen de la geología de la hoja Cuernavaca, estados de Morelos, México, Guerrero y Puebla: México, Universidad Nacional Autónoma de México, Instituto de Geología, Carta Geológica de México, Serie de 1:100.000, 1 mapa con texto al reverso.

Garduño-Monroy, V. H., Corona-Chávez, P., Israde-Alcántara, I., Mennella, L., Arreygue, E., Bigioggero, B, Chiesa, S., 1999, Carta geológica del estado de Michoacán, escala 1:250,000: Morelia, Universidad Michoacana de San Nicolás de Hidalgo, 111 p., 1 mapa.

GEOLIMEX Working Group, 1994, Reflections from the subducting plate? First results of a Mexican geotraverse, en, Miller, H., Rosenfeld, U., Weber-Diefenbach, K. (eds.), 13. Symposium on Latin-America Geosciences: Zentralblatt für Geologie und Paläontologie, Teil 1. Allgemeine, Angewandte, Regionale und Historische Geologie, 1-2, 541-553.

González-Partida, E., Levresse, G., Carrillo-Chávez, A., Cheilletz, A., Gasquet, D., Jones, D., 2003, Paleocene adakite Au- Fe bearing rocks, Mezcala, Mexico: evidence from geochemical characteristics: Journal of Geochemical Exploration, 80(1), 25-40.

González-Ramos, A., Sánchez-Rojas, L.E., Mota-Mota, S., Arceo y Cabrilla, F.A., Onofre-Espinoza, L., Zárate-López,J., Soto-Araiza, R., 2001, Carta geológico-minera y geoquímica de la hoja Oaxaca. Escala 1:250.000 (E14-9): Pachuca, Hidalgo, Consejo de Recursos Minerales, 1 mapa.

Guerrero-García, J. C., 1975, Contributions to paleomagnetism and $\mathrm{Rb}-\mathrm{Sr}$ geochronology: Dallas, Texas, University of Texas, Tesis doctoral, $131 \mathrm{p}$. 
Guinta, G., Beccaluva, L., Coltorti, M., Sienna, F., Mortellaro, D., Cutrupia, D., 2002, The peri-Caribbean ophiolites: structure, tectono-magmatic significance and geodynamic implications: Caribbean Journal of Earth Sciences, 36, 1-20.

Guzmán-Speziale, M., Meneses-Rocha, J. J., 2000, The North AmericaCaribbean plate boundary west of the Motagua-Polochic fault system: a fault jog in southeastern Mexico: Journal of South American Earth Sciences, 13, 459-468

Hart, S. R., 1984, A large-scale isotope anomaly in the southern hemisphere mantle: Nature, 309, 753-757.

Hernández-Bernal, M. S., Morán-Zenteno, D. J., 1996, Origin of the Rio Verde batholith, southern Mexico, as inferred from is geochemical characteristics: International Geology Review, 38, 361-373.

Hernández-Romano, U., Aguilera-Franco, N., Martínez-Medrano, M., Barceló-Duarte, J., 1997, Guerrero-Morelos Platform drowning at the Cenomanian-Turonian boundary, Huitziltepec area, Guerrero state, southern Mexico: Cretaceous Research, 18, 661-686.

Herrmann, U. R., Nelson, B. K., Ratschbacher, L., 1994, The origin of a terrane: $\mathrm{U} / \mathrm{Pb}$ zircon geochronology and tectonic evolution of the Xolapa complex (southern Mexico): Tectonics, 13, 455-474.

Jansma, P. E., Lang, R., 1997, The Arcelia graben: new evidence for Oligocene Basin and Range extension in southern Mexico: Geology, $25,455-458$.

Jording, A., Ferrari, L., Arzate, J., Jödicke, H., 2000, Crustal variations and terrane boundaries in southern Mexico as imaged by magnetotelluric transfer functions: Tectonophysics, 327, 1-13.

Kemp, A. I. S., Hawkesworth, C. J., 2004, Granitic perspectives on generation and secular evolution of the continental crust, en Rudnick, $\mathrm{R}$ L. (ed.), Treatise on geochemistry, volume 3, The crust: Amsterdam, Elsevier, 349-410.

Keppie, J. D., 2004, Terranes of Mexico revisited: 1.3 billion year odyssey: International Geology Review, 46, 765-794.

Keppie, J. D., Morán-Zenteno, D. J., 2005, Tectonic implications of alternative Cenozoic reconstructions for southern Mexico and the Chortis Block: International Geology Review, 47, 473-491.

Kratzeisen, M. J., Frank, M. M., Negendank, J. F. W., Boehnel, H., Terrell, D., 1991, The continental margin of southern Mexicotectonic evolution during the Tertiary: Zentralblatt für Geologie und Paläontologie, Teil I. Allgemeine, Angewandte, Regionale und Historische Geologie, 6, 1545-1555.

Lang, H. R., Frerichs, W. E., 1998, New planktic foraminiferal data documenting Conician age for Laramide orogeny onset and paleoceanography in southern Mexico: Journal of Geology, 106, 635-640.

Lang, H. R., Barros, J. A., Cabral-Cano, E., Draper, G., Harrison, C. G. A., Jansma, P. E., Johnson, C. A., 1996, Terrane deletion in northern Guerrero state: Geofísica Internacional, 35, 349 - 359.

Levresse, G., González-Partida, E., Carrillo-Chávez, A., Tritlla, J., Camprubí, A., Cheilletz, A., Gasquet, D., Deloule, E. 2004 Petrology, $\mathrm{U} / \mathrm{Pb}$ dating and $(\mathrm{C}-\mathrm{O})$ stable isotope constraints on the source and evolution of the adakite-related Mezcala Fe-Au skarn district, Guerrero, México: Mineralium Deposita, 39, 301-312.

López-Infanzón, M, Grajales-Nishimura, M., 1984, Edades de K-Ar de rocas ígneas y metamórficas del estado de Guerrero, en VII Convención Nacional, Resúmenes: México, Sociedad Geológica Mexicana, 215.

Malfait, B. T., Dinkelman, M. G., 1972, Circum-Caribbean tectonic and igneous activity and evolution of the Caribbean plate: Geological Society of America Bulletin, 83, 251-272.

Mammerickx, J., Klitgord, K. D., 1982, Northern east Pacific rise: evolution from 25 m.y. B.P. to the present: Journal of Geophysical Research, 87(B8), 6751-6759.

Martiny, B., Martínez-Serrano, R., Morán-Zenteno, D. J., Macías-Romo, C., Ayuso, R., 2000, Stratigraphy, geochemistry and tectonic significance of the Oligocene magmatic rocks in western Oaxaca, southern Mexico: Tectonophysics, 318, 71-98.

Martiny, B., Silva-Romo, G., Morán-Zenteno, D.J., 2002, Tertiary faulting and relationship with Eocene-Oligocene volcanism in western Oaxaca, southern Mexico, en 98th Annual Meeting, Cordilleran Section: Geological Society of America, Abstracts with Programs,
34(5) A-97.

Meneses-Rocha, J. J., 1991, Tectonic development of the Ixtapa graben, Chiapas, Mexico: Austin, Texas, University of Texas, Tesis doctoral, $120 \mathrm{p}$.

Meneses-Rocha, J. J., Rodríguez-Figueroa, D., Tóriz-Gama, J., BandaHernández, J., Hernández de la Fuente, R., Valdivieso-Ramos, V., 1996, Excursión geológica al cinturón plegado y cabalgado de Zongolica: México, Petróleos Mexicanos, Exploración y Producción, Reporte interno.

Meneses-Rocha, J. J., Monroy-Audelo, M. E., Gómez-Chavarria, J. C., 1994, Bosquejo paleogeográfico y tectónico del sur de México durante el Mesozoico: Boletín de la Asociación Mexicana de Geólogos Petroleros, 44, 1845.

Mercier de Lépinay, B., Michaud, F., Calmus, T., Burgois, J., Poupeau, G., Saint Marc, P., 1997, Large Neogene subsidence event along the middle America Trench off Mexico $\left(18^{\circ} \mathrm{N}-19^{\circ} \mathrm{N}\right)$ : evidence from submersible observations: Geology, 25, 387-390.

Meschede, M., Frisch, W., Herrmann, U. R., Ratschbacher, L., 1996, Stress transmission across an active plate boundary: an example from southern Mexico: Tectonophysics, 266, 81-100.

Meza-Figueroa, D., Valencia-Moreno M, V., Valencia, V. A., OchoaLandín, L., Pérez-Segura, E., Díaz-Salgado, C., 2003, Major and trace element geochemistry and 40Ar/39Ar geochronology of Laramide plutons associated with gold-bearing Fe skarn deposits in Guerrero state, southern Mexico: Journal of South American Earth Sciences, 16, 205-217.

Monod, O., Faure, M., Thieblemont, D., 1994, Guerrero terrane of Mexico; its role in the southern Cordillera from new geochemical data. Comment: Geology, 22(4) 477.

Monroy, M., Sosa, A., 1984, Geología de la Sierra del Tentzo, Puebla, borde norte del terreno Mixteco: Boletín de la Sociedad Geológica Mexicana, 45, 43-72.

Montiel-Escobar, J.E., Segura de la Teja, M.A., Estrada-Rodarte, G., Cruz-López, D., Rosales-Franco, E., 2000, Carta geológica-minera de la hoja Ciudad Altamirano. Escala 1:250.000 (E14-4): Pachuca, Hidalgo, Consejo de Recursos Minerales, 1 mapa.

Morán-Zenteno, D. J., 1992, Investigaciones isotópicas de Rb-Sr y Sm$\mathrm{Nd}$ en rocas cristalinas de la región Tierra Colorada - Acapulco - Cruz Grande, estado de Guerrero: México, Universidad Nacional Autónoma de México, Colegio de Ciencias y Humanidades, Unidad Académica de los Ciclos Profesional y de Posgrado, Instituto de Geofísica, Tesis doctoral, 186 p.

Morán-Zenteno, D. J., Corona-Chávez, P., Tolson, G., 1996, Uplift and subduction erosion in southwestern Mexico since the Oligocene: pluton geobarometry constraints: Earth and Planetary Science Letters, 141, 51-65.

Morán-Zenteno, D. J., Tolson, G., Martínez-Serrano, R. G., Martiny, B., Schaaf, P., Silva-Romo, G., Macías-Romo, C., Alba-Aldave, L., Hernández-Bernal, M. S., Solís-Pichardo, G. N., 1999, Tertiary arcmagmatism of the Sierra Madre del Sur, Mexico, and its transition to the volcanic activity of the Trans-Mexican Volcanic Belt: Journal of South American Earth Sciences, 12, 513 - 535.

Morán-Zenteno, D. J., Alba-Aldave, L. A., Solé, J., Iriondo, A., 2004, A major resurgent caldera in southern Mexico: the source of the late Eocene Tilzapotla ignimbrite: Journal of Volcanology and Geothermal Research, 136, 97-119.

Mossman, R.W., Viniegra, F., 1976, Complex fault structures in Veracruz Province of Mexico, The Association of Petroleum Geologists Bulletin, 60, 379-388.

Nava, A., Núñez-Cornú, F., Córdoba, D., Mena, M., Ansorge, J., González, J., Rodríguez, M., Banda, E., Mueller, S., Udías, A., García-García, M., Calderón, G., 1988, Structure of the middle America trench in Oaxaca, México: Tectonophysics, 154, 241-251.

NAVDAT, 2005, The Western North American Volcanic and Intrusive Rock Database: National Science Foundation; China Lake Naval Air, Weapons Station, Geothermal Program Office, http://navdat. geongrid.org. (Consulta de mayo del 2005).

Nieto-Samaniego, Á. F., Alaniz-Álvarez, S. A., Ortega-Gutiérrez, F., 1995, Estructura interna de la falla de Oaxaca (México) e influencia de las anisotropías litológicas durante su actividad cenozoica: Revista 
Mexicana de Ciencias Geológicas, 12, 1-8.

Nieto-Samaniego, Á. F., Ferrari, L., Alaniz-Álvarez, S. A., LabartheHernández, G., Rosas-Elguera, R., 1999, Variation of Cenozoic extension and volcanism across the southern Sierra Madre Occidental volcanic province, México: Geological Society of America Bulletin, 111, 347-363.

Nieto-Samaniego, A. F., Alaniz-Álvarez, S. A., Silva-Romo, G., EguizaCastro, M. H., Mendoza-Rosales, C. C., 2006, Latest Cretaceous to Miocene deformation events in the eastern Sierra Madre del Sur, Mexico, inferred from the geometry and age of major structures: Geological Society of America Bulletin, 118, 1868-1882S.

Ortega-Gutiérrez F., 1980, Rocas volcánicas del Maestrichtiano en el área de San Juan Tetelcingo, estado de Guerrero, en V Convención Geológica Nacional, Libro guía de la excursión geológica a la parte central de la cuenca del alto Río Balsas, estados de Guerrero y Puebla: México, Sociedad Geológica Mexicana, 34-38.

Ortega-Gutiérrez, F., 1981, Metamorphic belts of southern Mexico and their tectonic significance: Geofísica Internacional, 20, 177-202.

Ortega-Gutiérrez, F., Mitre-Salazar, L. M., Roldán-Quintana, J., ArandaGómez, J. J., Morán-Zenteno, D. J., Alaniz-Álvarez, S. A., NietoSamaniego, Á. F., 1992, Carta geológica de la República Mexicana, quinta edición escala 1:2.000,000: México, D. F., Universidad Nacional Autónoma de México, Instituto de Geología; Secretaría de Energía, Minas e Industria Paraestatal, Consejo de Recursos Minerales, 1 mapa

Pantoja-Alor, J., 1986, Siete edades geocronométricas cenozoicas de la cuenca media del Río Balsas, en Primer Simposio Geología Regional de México, Resúmenes: México, D.F., Universidad Nacional Autónoma de México, Instituto de Geología, 60-61.

Pardo, M., Suárez, G., 1995, Shape of the subducted Rivera and Cocos plates in southern Mexico: seismic and tectonic implications: Journal of Geophysical Research, 100(B7), 2357-12373.

Pasquaré, G., Ferrari, L., Garduño, V. H., Tibaldi, A., Vezzoli, L., 1991, Geology of the central sector of Mexican Volcanic Belt, states of Guanajuato and Michoacán: Boulder, Colorado, Geological Society of America, Map and Chart Series, MCH 072, 1 mapa, 22 p.

Pindell, J. L., Barret, S. F., 1990, Geological evolution of the Caribbean region; a plate perspective, in Dengo, G., Case, J. E. (eds.), The Caribbean region: Boulder, Colorado, Geological Society of America, The Geology of North America, H, 405-432.

Pindell, J. L., Cande, S. C. W., Pitman, W. C., Rowley, D. B., Dewey, J. F., Lebrecque, J., Haxby, W., 1988, A plate-kinematic framework for models of Caribbean evolution: Tectonophysics, 155, 121-138.

Pitcher, W. S., 1993, The nature and origin of granite: London, Blackie Academic \& Professional, $321 \mathrm{p}$.

Ponce, L., Gaulon, R., Suárez, G., Lomas, E., 1992, Geometry and state of stress of the downgoing Cocos plate in the Isthmus of Tehuantepec, Mexico: Geophysical Research Letters, 19, 773-776.

Ratschbacher, L., Riller, U., Meschede, M., Herrmann, U., Frisch, W., 1991, Second look at suspect terranes in southern Mexico: Geology, 19, 1233-1236.

Riller, U., Ratschbacher, L., Frisch, W., 1993, Left-lateral transtension along the Tierra Colorada deformation zone, northern margin of the Xolapa magmatic arc of southern Mexico: Journal of South American Earth Sciences, 5, 237-249.

Rivera-Carranza, E., Tejada-Segura, M.A., Miranda-Huerta, A., LemusBustos, O., Motolinía-García, O., León-Ayala, V., MoctezumaSalgado, M.D., 1998, Carta geológico-minera de la hoja Cuernavaca. Escala 1:250.000 (E14-5): Pachuca, Hidalgo, Consejo de Recursos Minerales, 1 mapa.

Robinson, K. L., Gastil, R. G., Campa, M. F., Ramírez-Espinosa, J., 1989, Geochronology of basement and metasedimentary rocks in southern Mexico and their relation to metasedimentary rocks in Peninsular California: Geological Society of America, Abstracts with Programs, 21(5), 135.

Rosencrantz, E., Sclater, J. G., 1986, Depth and age in the Cayman trough: Earth and Planetary Science Letters, 79, 133-144.

Ross, M., Scotese, C. R., 1988, A hierarchical tectonic model of the Gulf of Mexico and Caribbean region: Tectonophysics, 155, 139-168.

Saleeby, J., 2003, Segmentation of the Laramide slab - evidence from the southern Sierra Nevada region: Geological Society of America Bulletin, 115, 655-668.

Salinas-Prieto, J. C., Monod, O., Faure, M., 2000, Ductile deformations of opposite vergence in the eastern part of the Guerrero terrane (SW Mexico): Journal of South American Earth Sciences, 13, 389-402.

Sánchez-Barreda, L. A., 1981, Geologic evolution of the continental margin of the Gulf of Tehuantepec in southern Mexico: Austin, Texas, University of Texas, Tesis doctoral, $192 \mathrm{p}$.

Schaaf, P., 1990, Isotopengeochemishe untersuchugen an granitoiden gesteinen eines aktiven kontinentalrandes. Alter und herkufen der tiefengesteinskomplexe an der pazifikküste Mexikos zwischen Puerto Vallarta und Acapulco: München, Facultät fur Geowissenschaten der Ludwig-Maximilians Universität, Tesis doctoral, $202 \mathrm{p}$.

Schaaf, P., Morán-Zenteno, D., Hernández-Bernal, M. S., Solís-Pichardo, G., Tolson, G., Köhler H., 1995, Paleogene continental margin truncation in southwestern Mexico: geochronological evidence: Tectonics, 14, 1339-1350.

Sedlock, R. L., Ortega-Gutiérrez, F., Speed, C., 1993, Tectonostratigraphic terranes and tectonic evolution of Mexico: Boulder, Colorado, Geological Society of America, Special Paper, 278, 153 p.

Silva-Romo, G., Martiny, B., Mendoza-Rosales, C., 2001, Formación de cuencas continentales en el sur de México y su cronología respecto al desplazamiento del bloque de Chortis, en XI Congreso Latinoamericano de Geología, III Congreso Uruguayo de Geología, Memoria: Montevideo, Uruguay, 9.

Smith, R. D., Cameron, K. L., McDowell, F. W., Niemeyer, S., Sampson, D. E., 1996, Generation of voluminous silicic magmas and formation of mid-Cenozoic crust beneath north-central Mexico: evidence from ignimbrites, associated lavas, deep crustal granulites and mantle pyroxenites: Contributions to Mineralogy and Petrology, $123,375-389$

Stacey, J. S., Kramers, J. D., 1975, Approximation of terrestrial south lead isotope evolution by a two-stage model: Earth and Planetary Science Letters, 26, 207-221.

Tolson, G., Solís-Pichardo, G., Morán-Zenteno, D. J., Victoria-Morales, A., Hernández-Treviño, J. T., 1993, Naturaleza petrográfica y estructural de las rocas cristalinas de 1a zona de contacto entre los terrenos Xolapa y Oaxaca, región de Santa María Huatulco, Oaxaca, en Delgado-Argote, L., Martín-Barajas, A. (eds.), Contribuciones a la tectónica del occidente de México: Ensenada, B. C., Unión Geofísica Mexicana, Monografía, 1, 327-349.

Tolson-Jones, G., 1998, Deformación, exhumación y neotectónica de la margen continental de Oaxaca: datos estructurales, petrológicos y geotermobarométricos: México, Universidad Nacional Autónoma de México, Colegio de Ciencias y Humanidades, Unidad Académica de los Ciclos Profesionales y de Posgrado, Posgrado en Geofísica, Tesis doctoral, $98 \mathrm{p}$.

Tritlla, J., Camprubí, A., Centeno-García, E., Corona-Esquivel, R., Sánchez-Martínez, S., Gasca-Durán, A., Cienfuegos-Alvarado, E., Morales-Puente, P., 2003, Estructura y edad del depósito de hierro de Peña Colorada (Colima): un posible equivalente fanerozoico de los depósitos de tipo IOCG: Revista Mexicana de Ciencias Geológicas, 20, 182-201.

Urrutia-Fucugauchi, J., Flores-Ruiz, J. H., 1996, Bouguer gravity anomalies and regional crustal structure in central Mexico: International Geology Review, 38, 176-194.

Urrutia-Fucugauchi, J., Ferrusquía-Villafranca, I., 2001, Paleomagnetic results for the Middle-Miocene continental Suchilquitongo Formation, Valley of Oaxaca, southeastern Mexico: Geofísica Internacional, 40, 191-206.

Valdes, C. M., Mooney, W., Singh, K., Meyer, S., Lomnitz, C., Luetgert, J., Hesley, C., Lewis, B., Mena, M., 1986, Crustal structure of Oaxaca, Mexico, from seismic refraction measurements: Bulletin of the Seismological Society of America, 76, 547-563.

Manuscrito recibido: Octubre 22, 2004

Manuscrito corregido recibido: Mayo 25, 2005

Manuscrito aceptado: Mayo 30, 2005 